\title{
Identification of crucial miRNAs and lncRNAs for ossification of ligamentum flavum
}

\author{
DALIANG KONG ${ }^{1}$, QIHENG ZHAO ${ }^{1}$, WENPING LIU $^{2}$ and FEI WANG ${ }^{1}$ \\ ${ }^{1}$ Department of Orthopedics, China-Japan Union Hospital, Jilin University, Changchun, Jilin 130033; \\ ${ }^{2}$ Department of Neurology, Second Hospital of Jilin University, Changchun, Jilin 130031, P.R. China
}

Received October 17, 2018; Accepted April 10, 2019

DOI: $10.3892 / \mathrm{mmr} .2019 .10377$

\begin{abstract}
The present study aimed to screen crucial micro (mi)RNAs and long non-coding (lnc)RNAs involved in the development of ossification of ligamentum flavum (OLF) based on the miRNA-mRNA and lncRNA-miRNA-mRNA competing endogenous (ce)RNA regulatory network analyses, which are rarely reported. The differentially expressed genes (DEGs), differentially expressed lncRNAs (DELs) and differentially expressed miRNAs (DEMs) between 4 OLF and 4 healthy controls were identified using two microarray datasets GSE106253 and GSE106256 collected from the Gene Expression Omnibus database. A protein-protein interaction (PPI) network was constructed, followed by calculation of topological characteristics and sub-module analysis in order to obtain hub DEGs. The miRNA-mRNA and IncRNA-miRNA networks that were established based on their interaction pairs, obtained from miRwalk and starBase databases, respectively, were integrated to form the ceRNA network. The underlying functions of mRNAs were predicted using the Database for Annotation, Visualization and Integrated Discovery (DAVID). The present study screened 828 DEGs, 119 DELs and 81 DEMs between OLF and controls. PPI network and module analyses identified interleukin (IL)10, adenylate cyclase (ADCY)5, suppressor of cytokine signaling (SOCS)3, G protein subunit gamma (GNG) 4, collagen type II $\alpha 1$ chain (COL2A1) and collagen type XIII $\alpha 1$ chain (COL13A1) as hub genes. The miRNA-mRNA network analysis demonstrated IL10 could be regulated by miR-210-3p, while COL13A1 and COL2A1 could be modulated by miR-329-3p and miR-222-5p, respectively. IncRNA-miRNA-mRNA ceRNA network analysis identified that small nucleolar RNA host gene 16-hsa-miR-196a-5p-SOCS3, ankyrin repeat and SOCS
\end{abstract}

Correspondence to: Dr Fei Wang, Department of Orthopedics, China-Japan Union Hospital, Jilin University, 126 Xiantai Street, Changchun, Jilin 130033, P.R. China

E-mail: feiwang1017@yeah.net

Key words: ossification, ligamentum flavum, long non-coding RNAs, microRNAs, competing endogenous RNAs, inflammation, osteogenic differentiation box containing 16-AS1-hsa-miR-379-5p-GNG4, nuclear enriched abundant transcript 1-has-miR-181b-5p-ADCY5, rhophilin 1-AS1-hsa-miR-299-3p-WNT7B interaction axes may be crucial. DAVID analysis predicted IL10, ADCY5, GNG4 and SOCS3 were involved in 'adaptive immune response', 'Chemokine signaling pathway' and 'regulation of apoptosis' processes, while COL2A1, COL13A1 and WNT7B may be ossification related. In conclusion, the identification of these crucial miRNAs and lncRNAs may be conducive for explaining the pathogenesis of OLF and provide certain natural, endogenous and nontoxic drug targets for the treatment of OLF.

\section{Introduction}

Ossification of ligamentum flavum (OLF) is a relatively common spinal disorder in Eastern Asian countries, with an estimated prevalence of $63.9 \%$ in Chinese (1), 36\% in Japanese (2) and $16.9 \%$ in Korean (3) populations. OLF is characterized by ectopic bone formation in the spinal ligaments and ligamentous tissue hyperplasia (4) that cause spinal canal narrowing and result in the development of myelopathy and radiculopathy $(5,6)$. Surgery is the predominant treatment option for OLF; however, the difficulty of surgery and a relatively high risk of complications have to be taken into consideration (7). Therefore, it is necessary to develop more effective, convenient and safe approaches for the treatment of OLF; an improved understanding of its molecular mechanisms may provide insight.

Although the pathogenesis of OLF remains to be elucidated, abnormal expression of osteogenic differentiation and cell proliferation related genes in LF cells may serve important roles (8). The mRNA levels of osteogenic markers [alkaline phosphatase (ALP), runt-related transcription factor 2, osterix and osteopontin)] in addition to signaling pathway genes [bone morphogenetic proteins (BMPs), Wnt/ $\beta$-catenin and Notch] $(9,10)$, were identified to be higher in patients with OLF compared with non-OLF subjects. Recombinant BMP2 or BMP14 [also known as growth/differentiation factor (GDF) 5] modification induced the osteoblastic differentiation of LF cells and promoted bone nodule formation, finally triggering neurological impairment in rat models $(11,12)$, while downregulation of Notch 2 ameliorated the processes (10). In addition to accelerating osteoblast differentiation via osterix, 

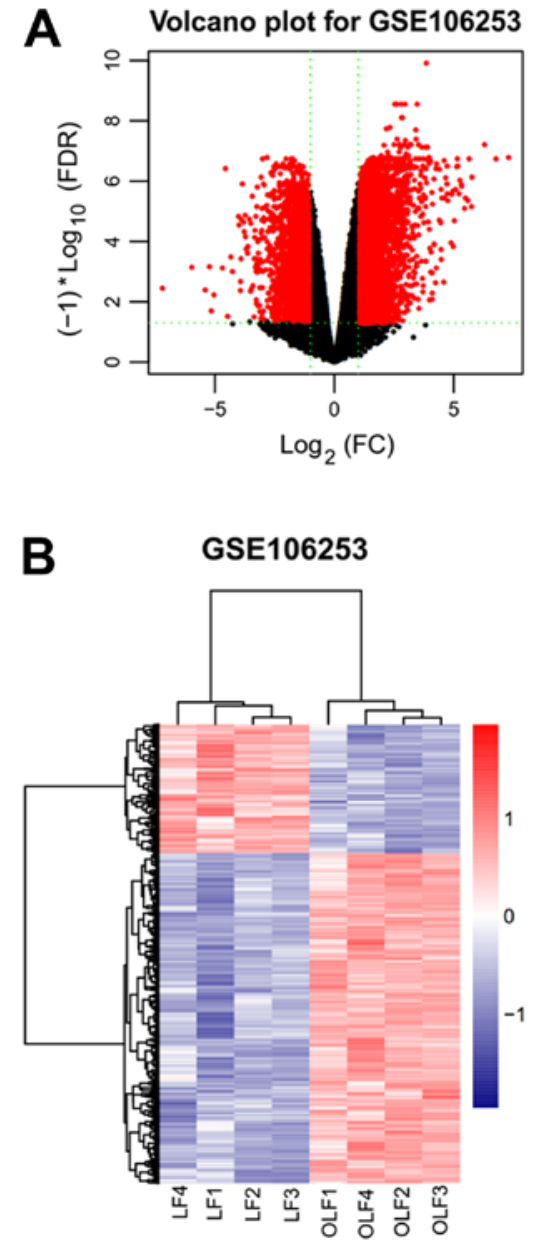

Figure 1. Expression pattern of mRNA, lncRNAs and miRNAs in patients with OLF and healthy individuals. (A) Volcano plot shows the number and distribution of mRNAs and lncRNAs in GSE106253, and miRNAs in GSE106256. Red dots indicated differentially expressed genes; black dots indicated no significant difference in expression. (B) Heat map showing differentially expressed mRNAs and lncRNAs in GSE106253, and miRNAs in GSE106256 when comparing OLF with the control. Red and blue denoted high and low expression, respectively. IncRNA, long non-coding RNA; miRNA, microRNA; OLF, ossification of ligamentum flavum; FC, fold change; FDR, false discovery rate.

highly expressed pro-inflammatory cytokines [tumor necrosis factor (TNF)- $\alpha$, interleukin (IL)-1a and IL-6] appear to stimulate cell proliferation and tissue hypertrophy by upregulating cyclin D1 and c-Myc in OLF (13-15). Therefore, targeted regulation of these genes may be potential strategies for the treatment of OLF.

A potential way to endogenously regulate the expression levels of target mRNAs is through microRNAs (miRNAs/miRs) that bind to the 3'-untranslated regions of target genes and subsequently mediate their degradation or translation inhibition (16). Therefore, researchers are exploring the crucial miRNAs that regulate the expression of osteogenic differentiation related genes in OLF. miR-132-3p and miR-615-3p have been demonstrated to be downregulated during osteogenic differentiation of LF cells $(17,18)$. Overexpression of miR-615-3p by its mimics suppressed the osteogenic differentiation of LF cells by reducing the expression of GDF5 (17). miR-199b-5p and miR-487b-3p were reported to inhibit osteogenic differentiation in LF cells by downregulating Notch and Wnt signaling pathway genes, respectively $(19,20)$. However, the OLF-related miRNAs have rarely been reported and the inflammation-associated miRNAs in OLF have not been identified.
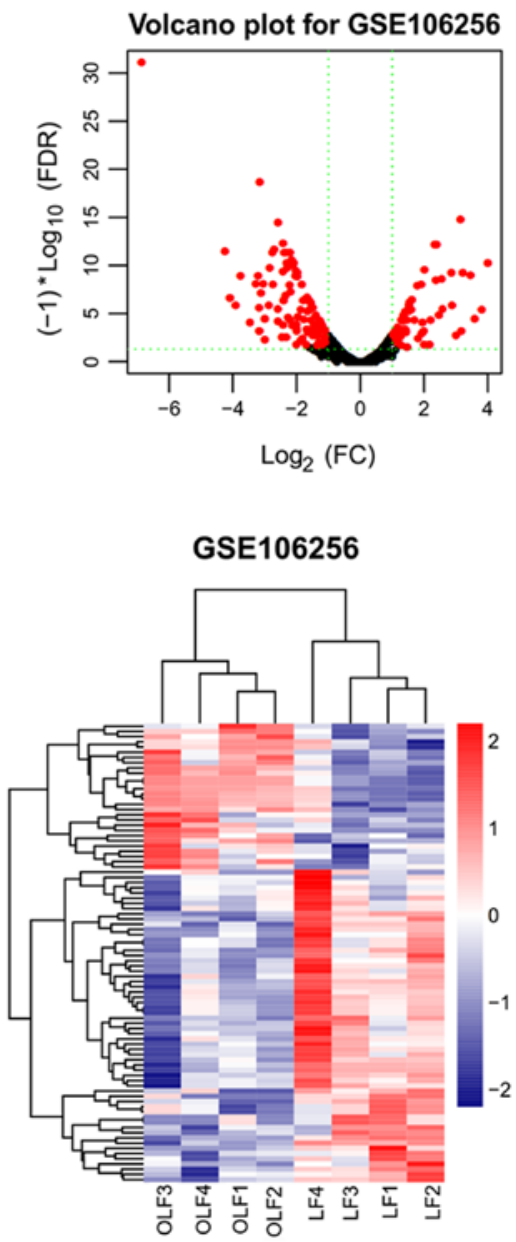

In addition to miRNAs, long non-coding RNAs (lncRNAs) are considered to be crucial in regulating the expression of genes. IncRNAs can competitively bind to miRNAs through their miRNA response elements and influence the regulation of miRNAs for mRNAs, which is called the competing endogenous RNA (ceRNA) hypothesis (21). Therefore, IncRNAs may also be important targets for the treatment of OLF. However, OLF-related IncRNAs are rarely reported, with the exception of the a previous study by Han et al (22).

The aim of the present study was to use the datasets uploaded by Han et al (22) to further identify novel miRNAs and crucial lncRNAs for OLF based on the miRNA-mRNA and IncRNA-miRNA-mRNA ceRNA regulatory networks. The findings may provide insight for underlying therapeutic strategies for OLF by changing the expression levels of miRNAs and lncRNAs, which could in turn regulate the target genes.

\section{Materials and methods}

Data sources. A total of two datasets under accession numbers GSE106253 and GSE106256 (22) were downloaded from the Gene Expression Omnibus (GEO) database (http://www.ncbi. 
Table I. Top 10 upregulated and downregulated differentially expressed genes, lncRNAs and miRNAs.

A, Upregulated

\begin{tabular}{|c|c|c|c|c|c|c|c|c|}
\hline miRNA & FDR & $\log \mathrm{FC}$ & lncRNA & FDR & $\log \mathrm{FC}$ & mRNA & FDR & $\log \mathrm{FC}$ \\
\hline hsa-miR-653-3p & $8.30 \times 10^{4}$ & 4.00 & LINC01549 & $2.31 \times 10^{2}$ & 5.50 & PKIB & $6.80 \times 10^{3}$ & 6.29 \\
\hline hsa-miR-489-3p & $2.33 \times 10^{2}$ & 3.81 & CLSTN2-AS1 & $1.45 \times 10^{2}$ & 4.678 & AMTN & $2.95 \times 10^{2}$ & 5.47 \\
\hline hsa-miR-508-3p & $4.51 \times 10^{2}$ & 3.59 & LINC00347 & $2.04 \times 10^{2}$ & 4.09 & WISP3 & $1.77 \times 10^{2}$ & 5.34 \\
\hline hsa-miR-4683 & $1.95 \times 10^{3}$ & 3.46 & LINC02203 & $7.15 \times 10^{3}$ & 3.84 & ADCYAP1 & $1.70 \times 10^{2}$ & 5.32 \\
\hline hsa-miR-138-5p & $1.70 \times 10^{3}$ & 3.22 & LINC01508 & $4.07 \times 10^{2}$ & 3.75 & COL9A1 & $1.11 \times 10^{2}$ & 5.17 \\
\hline hsa-miR-653-5p & $3.54 \times 10^{5}$ & 3.14 & WASIR2 & $1.07 \times 10^{2}$ & 3.22 & SERPINA11 & $1.13 \mathrm{E}-02$ & 5.13 \\
\hline hsa-miR-4473 & $1.72 \times 10^{2}$ & 2.88 & LINC01440 & $9.83 \times 10^{3}$ & 3.18 & CLEC3A & $1.89 \times 10^{2}$ & 5.08 \\
\hline hsa-miR-483-3p & $1.65 \times 10^{3}$ & 2.86 & LINC02249 & $1.12 \times 10^{2}$ & 3.14 & SLITRK6 & $3.88 \times 10^{2}$ & 5.03 \\
\hline hsa-miR-181b-3p & $2.28 \times 10^{2}$ & 2.58 & DSG1-AS1 & $2.27 \times 10^{2}$ & 3.07 & ZMAT4 & $2.11 \times 10^{2}$ & 4.92 \\
\hline
\end{tabular}

B, Downregulated

\begin{tabular}{|c|c|c|c|c|c|c|c|c|}
\hline miRNA & FDR & $\log \mathrm{FC}$ & lncRNA & FDR & $\log \mathrm{FC}$ & mRNA & FDR & $\log \mathrm{FC}$ \\
\hline hsa-miR-495-3p & $2.41 \times 10^{6}$ & -3.16 & LINC01706 & $2.24 \times 10^{2}$ & 3.06 & ITIH6 & $1.11 \times 10^{2}$ & 4.73 \\
\hline hsa-miR-495-3p & $2.41 \times 10^{6}$ & -3.16 & LINC00601 & $3.88 \times 10^{2}$ & -1.839 & FAM3B & $1.43 \times 10^{2}$ & -3.06 \\
\hline hsa-miR-377-5p & $2.07 \times 10^{2}$ & -3.18 & LINC01730 & $4.24 \times 10^{2}$ & -1.85 & ZIC3 & $1.79 \times 10^{2}$ & -3.22 \\
\hline hsa-miR-551b-3p & $2.04 \times 10^{3}$ & -3.21 & FAM230B & $2.48 \times 10^{2}$ & -1.88 & ANGPTL4 & $4.87 \times 10^{2}$ & -3.31 \\
\hline hsa-miR-369-5p & $3.61 \times 10^{3}$ & -3.28 & FLG-AS1 & $1.63 \times 10^{2}$ & -1.93 & SOCS3 & $2.20 \times 10^{2}$ & -3.43 \\
\hline hsa-miR-1185-1-3p & $2.11 \times 10^{3}$ & 3.76 & SNHG16 & $2.56 \times 10^{2}$ & -2.06 & GPT & $4.11 \times 10^{2}$ & -3.49 \\
\hline hsa-miR-539-3p & $1.72 \times 10^{2}$ & -3.91 & LINC01615 & $2.14 \times 10^{2}$ & -2.08 & ADAMTS4 & $3.43 \times 10^{2}$ & -3.73 \\
\hline hsa-miR-222-5p & $1.02 \times 10^{2}$ & -4.09 & HIPK1-AS1 & $1.98 \times 10^{2}$ & -2.30 & GPD1 & $4.02 \times 10^{2}$ & -3.79 \\
\hline hsa-miR-412-5p & $1.02 \times 10^{2}$ & -4.09 & LINC01485 & $3.95 \times 10^{2}$ & -2.53 & FAM71A & $1.67 \times 10^{2}$ & -3.84 \\
\hline hsa-miR-4443 & $3.53 \times 10^{4}$ & -4.25 & MEG3 & $2.80 \times 10^{2}$ & -2.53 & SAA1 & $3.77 \times 10^{2}$ & -3.89 \\
\hline hsa-miR-122-5p & $4.40 \times 10^{10}$ & -6.87 & VPS9D1-AS1 & $1.30 \times 10^{2}$ & -2.65 & CCL2 & $4.29 \times 10^{2}$ & -3.99 \\
\hline
\end{tabular}

lncRNA, long non-coding RNA; miRNA/miR, microRNA; FDR, false discovery rate; FC, fold change.

nlm.nih.gov/geo/) on July 2018. The GSE106253 dataset was analyzed to examine the mRNA and lncRNA expression profiles using the microarray technique (platform: GPL21827, Agilent-079487 Arraystar Human LncRNA microarray V4). Then, GSE106256 dataset was analyzed to detect the miRNA expression profile using high throughput sequencing (platform: GPL18573, Illumina NextSeq 500). These two datasets contained the LF tissues from 4 patients with OLF and 4 healthy volunteers.

Data preprocessing and differential analysis. The raw TXT data were collected from the GEO database and preprocessed using the Linear Models for Microarray data (LIMMA) method (23) (version 3.34.0; http://www.bioconductor.org/packages/release/bioc/html/limma.html) in the Bioconductor $\mathrm{R}$ package (version 3.4.1; http://www.R-project.org/), including base-2 $\log$ arithmic $(\log 2)$ transformation to normalize the skewed distribution, followed by quantile normalization. For the GSE106253 microarray data, all the probe sequences downloaded from the annotation platform GPL21827 were aligned and compared with the human genome using Clustal W program (version 2; http://www.clustal.org/) (24) to obtain the expression levels of lncRNA and mRNAs.
The differentially expressed genes (DEGs), differentially expressed IncRNAs (DELs) and differentially expressed miRNAs (DEMs) between the patients with OLF and the healthy controls were identified using the LIMMA method (23). DEGs, DELs and DEMs were screened based on the statistical threshold of $\mid \log F C$ (fold change) $\mid>1$ and false discovery rates (FDR) $<0.05$. Two-way hierarchical clustering was performed using pheatmap R package (version: 1.0.8; https://cran.r-project. org/web/packages/pheatmap) based on Euclidean distance to render a heatmap of DEGs, DELs and DEMs.

Protein-protein interaction (PPI) network of DEGs. The DEGs were mapped to the Search Tool for the Retrieval of Interacting Genes (STRING; version 10.0; http://string db.org/) database (25) to acquire PPI pairs. Then, the PPI network was constructed using these PPI pairs and visualized using Cytoscape software (version 3.6.1; www.cytoscape. org/) (26). Topological features of each node (protein) in the PPI network, including degree [the number of edges (interactions) of a node] and betweenness (BC; the number of shortest paths that run through a node), were used to screen hub candidate markers that serve crucial roles in OLF using the CytoNCA plugin in Cytoscape software (http://apps. 
Table II. Top 30 genes ranked by topological characteristics.

\begin{tabular}{|c|c|c|c|c|c|}
\hline Genes & Degree & Genes & Betweenness centrality & Overlap & $\operatorname{LogFC}$ \\
\hline AKT1 & 31 & TBC1D10B & 1.0000 & VEGFA & -1.55 \\
\hline CTNNB1 & 30 & GPR153 & 1.0000 & BMP4 & 2.26 \\
\hline VEGFA & 29 & PLK3 & 1.0000 & CTNNB1 & -1.90 \\
\hline GNG4 & 26 & POLQ & 0.8333 & GNG4 & 3.49 \\
\hline ADCY5 & 26 & LMNA & 0.6667 & AKT1 & -1.27 \\
\hline CCL5 & 19 & SUN2 & 0.6667 & POTEJ & -1.94 \\
\hline IL10 & 19 & EXO1 & 0.5000 & SH3GL1 & -1.62 \\
\hline POTEJ & 16 & CTNNB1 & 0.2625 & IL10 & -2.29 \\
\hline SAA1 & 16 & AKT1 & 0.1816 & ICAM1 & 1.38 \\
\hline SOCS3 & 15 & VEGFA & 0.1401 & MYC & -1.19 \\
\hline NMU & 15 & GNA12 & 0.1294 & ADCY5 & -1.46 \\
\hline NMUR1 & 15 & ADCY5 & 0.1213 & SOCS3 & -3.43 \\
\hline ITGA4 & 15 & YWHAZ & 0.0964 & CCL5 & 1.38 \\
\hline MYC & 15 & POLR2D & 0.0957 & ITGA4 & 1.64 \\
\hline COL4A2 & 14 & IL10 & 0.0833 & & \\
\hline COL9A2 & 14 & ITGA4 & 0.0756 & & \\
\hline SH3GL1 & 14 & POTEJ & 0.0725 & & \\
\hline COL9A3 & 14 & GBF1 & 0.0718 & & \\
\hline CCL2 & 14 & MYC & 0.0693 & & \\
\hline ICAM1 & 14 & PLA2G4F & 0.0665 & & \\
\hline COL6A1 & 13 & SMG5 & 0.0639 & & \\
\hline COL10A1 & 13 & CTTN & 0.0580 & & \\
\hline BMP4 & 13 & PLD4 & 0.0550 & & \\
\hline COL6A2 & 13 & ICAM1 & 0.0545 & & \\
\hline COL9A1 & 13 & SOCS3 & 0.0539 & & \\
\hline CD40LG & 13 & RPS18 & 0.0536 & & \\
\hline COL2A1 & 13 & CCL5 & 0.0532 & & \\
\hline CRTAP & 12 & RBBP4 & 0.0510 & & \\
\hline KBTBD7 & 12 & GNG4 & 0.0492 & & \\
\hline COL22A1 & 12 & FZD9 & 0.0475 & & \\
\hline PPIB & 12 & BMP4 & 0.0457 & & \\
\hline NT5E & 12 & SH3GL1 & 0.0448 & & \\
\hline COL13A1 & 12 & FOXO3 & 0.0426 & & \\
\hline PIK3R3 & 12 & TBL2 & 0.0424 & & \\
\hline
\end{tabular}

FC, fold change.

cytoscape.org/apps/cytonca) (27). The Molecular Complex Detection (MCODE; version:1.4.2, http://apps.cytoscape. org/apps/mcode) (28) plugin of the Cytoscape software was applied to extract highly interconnected sub-modules from the overall PPI network.

DEMs-regulated lncRNAs and genes. The DEMs regulated target genes were predicted using the miRwalk database (version 2.0; http://www.zmf.umm.uni-heidelberg. de/apps/zmf/mirwalk2) (29). The DEMs regulated lncRNAs were predicted using the starBase database (version 2.0; http://starbase.sysu.edu.cn/index.php) (30). The target genes and lncRNAs of DEMs were respectively overlapped with the DEGs and DELs to obtain the DEM-DEG and
DEM-DEL interaction networks, which were visualized using Cytoscape software (26). Based on the common miRNAs, the DEM-DEG and DEM-DEL networks were integrated to form a DEL-DEM-DEG ceRNA network, which was also visualized using Cytoscape software (26).

Function enrichment analysis. Gene Ontology (GO; release 2018-10-01; http://www.geneontology.org) term and The Kyoto Encyclopedia of Genes and Genomes (KEGG; release 88.0; https://www.kegg.jp) pathway enrichment analyses were conducted for genes in each sub-module network and all regulatory networks using the Biological Networks Gene Ontology (BINGO; version 3.0.3; https://www.psb.ugent. be/cbd/papers/BiNGO/Home.html) and the Database for 
A

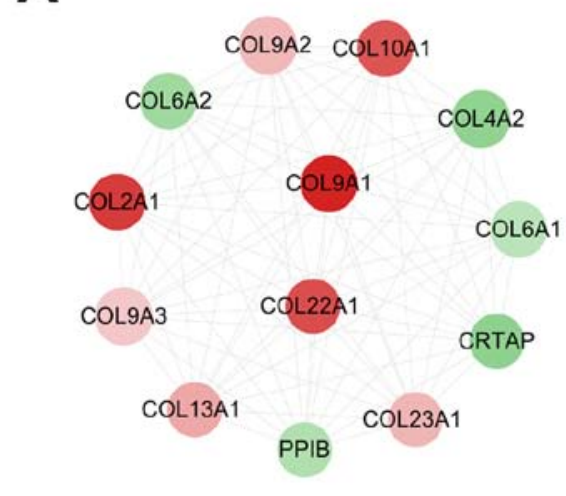

B

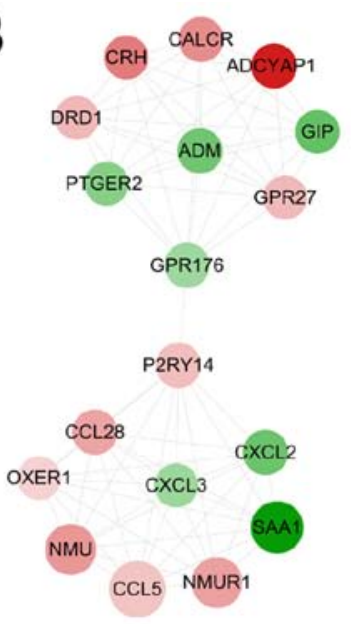

$\mathbf{F}$

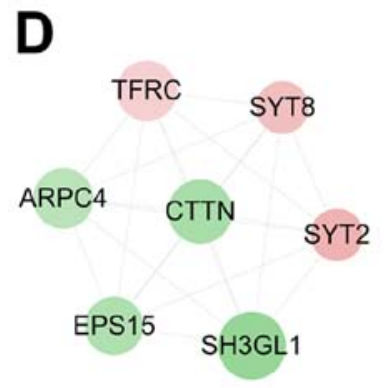

E

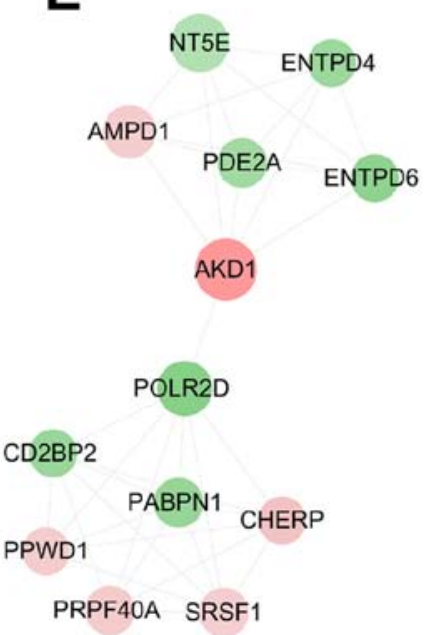

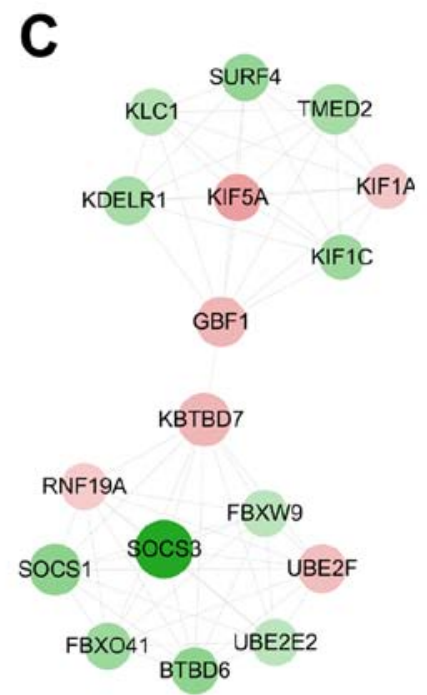

G

Figure 2. Modules extracted from the protein-protein interaction network. (A) Module 1, (B) module 2, (C) module 3, (D) module 4, (E) module 5, (F) module 6 and $(\mathrm{G})$ module 7 are presented. Red and green denoted upregulated and downregulated expression, respectively. The color depth is in proportion to differential levels. The size of the proteins is in proportion to the number of its interaction pairs.

Annotation, Visualization and Integrated Discovery (DAVID; version 6.8; http://david.abcc.ncifcrf.gov) (31) tools. $\mathrm{P}<0.05$ was considered to indicate a statistically significant difference.

\section{Results}

Expression pattern of $m R N A$, lncRNAs and miRNAs in OLF. Based on the cut-off criteria (FDR $<0.05$ and $\mid \log F C l>1$ ), a total of 828 DEGs (434 upregulated and 394 downregulated) and 119 DELs (94 upregulated and 25 downregulated) were identified in the gene chip GSE106253 (Fig. 1A); 81 DEMs, with 25 upregulated and 56 downregulated, were identified in the gene chip GSE106256 (Fig. 1A). The top 20 dys-regulated DEGs, DELs and DEMs are summarized in Table I. The hierarchically clustered heat map indicated that the DEGs and DELs in GSE106253 (Fig. 1B), and DEMs in GSE106256 (Fig. 1B) were well categorized into OLF and control groups.

DEGs interaction network construction. By searching the STRING database, 859 interaction pairs between DEGs were collected, which were used to create a PPI network, consisting of 372 nodes (168 upregulated and 204 downregulated; data not shown). A total of 14 nodes were identified and the top 30 genes were ranked following the calculation of the two topological features (the degree and BC), suggesting that 14 genes [vascular endothelial growth factor (VEGF) A, BMP4, catenin $\beta(\mathrm{CTNNB}) 1, \mathrm{G}$ protein subunit gamma (GNG) 4 , AKT serine/threonine kinase 1 (AKT1); POTE ankyrin domain family member (POTE) J, SH3 domain containing GRB2 like (SH3GL) 1, endophilin A2, IL10, intercellular adhesion molecule (ICAM) 1, MYC proto-oncogene (MYC), bHLH transcription factor, adenylate cyclase (ADCY) 5, suppressor of cytokine signaling (SOCS) 3, C-C motif chemokine ligand (CCL) 5 and integrin subunit $\alpha$ (ITGA) 4] may be hub genes in the PPI network (Table II). In addition, several collagen genes, including collagen type II $\alpha 1$ chain (COL2A1) and collagen type XIII $\alpha 1$ chain (COL13A1) may be also important for OLF, according to the degree ranking. A total of seven highly interconnected sub-modules were extracted from the PPI network using the MCODE algorithm (Fig. 2). Among them, 
Table III. Function enrichment for genes in the different modules.

\begin{tabular}{llll}
\hline A, M1 & & & \\
\hline GO-ID & P-value & \multicolumn{1}{c}{ Description } & \multicolumn{1}{c}{ Genes in test set } \\
\hline 48731 & $4.98 \times 10^{3}$ & System development & COL2A1, COL13A1, COL9A1, COL10A1, COL9A3, COL9A2 \\
48856 & $7.99 \times 10^{3}$ & Anatomical structure development & COL2A1, COL13A1, COL9A1, COL10A1, COL9A3, COL9A2 \\
1501 & $2.69 \times 10^{6}$ & Skeletal system development & COL2A1, COL13A1, COL9A1, COL10A1, COL9A2 \\
7155 & $1.08 \times 10^{4}$ & Cell adhesion & COL2A1, COL13A1, COL6A2, COL6A1, COL9A1 \\
22610 & $1.08 \times 10^{4}$ & Biological adhesion & COL2A1, COL13A1, COL6A2, COL6A1, COL9A1 \\
30198 & $5.74 \times 10^{5}$ & Extracellular matrix organization & COL2A1, COL4A2, COL6A2 \\
43062 & $2.12 \times 10^{4}$ & Extracellular structure organization & COL2A1, COL4A2, COL6A2 \\
16337 & $1.22 \times 10^{3}$ & Cell-cell adhesion & COL2A1, COL13A1, COL6A2 \\
\hline
\end{tabular}

$\mathrm{B}, \mathrm{M} 2$

\begin{tabular}{|c|c|c|c|}
\hline GO-ID & P-value & Description & Genes in test set \\
\hline 23052 & $6.96 \times 10^{7}$ & Signaling & $\begin{array}{l}\text { GPR27, P2RY14, PTGER2, ADM, GIP, ADCYAP1, GPR176, } \\
\text { CALCR, CCL5, NMU, CRH, OXER1, NMUR1, DRD1 }\end{array}$ \\
\hline 7166 & $7.13 \times 10^{7}$ & $\begin{array}{l}\text { Cell surface receptor linked } \\
\text { signaling pathway }\end{array}$ & $\begin{array}{l}\text { ADCYAP1, GPR176, CALCR, P2RY14, PTGER2, NMU, } \\
\text { OXER1, NMUR1, DRD1, GIP }\end{array}$ \\
\hline 23033 & $7.80 \times 10^{6}$ & Signaling pathway & $\begin{array}{l}\text { ADCYAP1, GPR176, CALCR, P2RY14, PTGER2, NMU, } \\
\text { OXER1, ADM, NMUR1, DRD1, GIP }\end{array}$ \\
\hline 23046 & $8.09 \times 10^{5}$ & Signaling process & $\begin{array}{l}\text { GPR27, GPR176, CALCR, CCL5, NMU, CRH, ADM, } \\
\text { NMUR1, DRD1, GIP }\end{array}$ \\
\hline 23060 & $8.09 \times 10^{5}$ & Signal transmission & $\begin{array}{l}\text { GPR27, GPR176, CALCR, CCL5, NMU, CRH, ADM, } \\
\text { NMUR1, DRD1, GIP }\end{array}$ \\
\hline 50896 & $2.69 \times 10^{4}$ & Response to stimulus & $\begin{array}{l}\text { CALCR, P2RY14, CCL5, NMU, SAA1, CRH, ADM, DRD1, } \\
\text { CXCL3, CXCL2, CCL28, GIP }\end{array}$ \\
\hline 65007 & $1.12 \times 10^{2}$ & Biological regulation & $\begin{array}{l}\text { GPR27, PTGER2, ADM, GIP, ADCYAP1, CALCR, CCL5, } \\
\text { NMU, SAA1, CRH, OXER1, NMUR1, DRD1, CCL28 }\end{array}$ \\
\hline 50794 & $1.32 \times 10^{2}$ & Regulation of cellular process & $\begin{array}{l}\text { GPR27, PTGER2, ADM, GIP, ADCYAP1, CALCR, CCL5, } \\
\text { NMU, SAA1, CRH, OXER1, NMUR1, DRD1 }\end{array}$ \\
\hline
\end{tabular}

C, M3

\begin{tabular}{|c|c|c|c|}
\hline GO-ID & P-value & Description & Genes in test set \\
\hline 7017 & $5.49 \times 10^{5}$ & Microtubule-based process & RNF19A, KIF5A, KIF1C, KIF1A \\
\hline 46627 & $1.03 \times 10^{4}$ & $\begin{array}{l}\text { Negative regulation of insulin } \\
\text { receptor signaling pathway }\end{array}$ & SOCS3, SOCS1 \\
\hline 7018 & $1.20 \times 10^{4}$ & Microtubule-based movement & KIF5A, KIF1C, KIF1A \\
\hline 46626 & $1.59 \times 10^{4}$ & $\begin{array}{l}\text { Regulation of insulin receptor } \\
\text { signaling pathway }\end{array}$ & SOCS3, SOCS1 \\
\hline 6890 & $1.59 \times 10^{4}$ & $\begin{array}{l}\text { Retrograde vesicle-mediated } \\
\text { transport, Golgi to ER }\end{array}$ & GBF1, KIF1C \\
\hline 32570 & $2.08 \times 10^{4}$ & Response to progesterone stimulus & SOCS3, SOCS1 \\
\hline 7259 & $4.46 \times 10^{4}$ & JAK-STAT cascade & SOCS3, SOCS1 \\
\hline 31100 & $4.72 \times 10^{4}$ & Organ regeneration & SOCS3, SOCS1 \\
\hline 32355 & $1.44 \times 10^{3}$ & Response to estradiol stimulus & SOCS3, SOCS1 \\
\hline 16192 & $1.52 \times 10^{3}$ & Vesicle-mediated transport & KDELR1, GBF1, KIF1C, TMED2 \\
\hline 51246 & $1.99 \times 10^{3}$ & $\begin{array}{l}\text { Regulation of protein metabolic } \\
\text { process }\end{array}$ & UBE2F, SOCS3, SOCS1, UBE2E2 \\
\hline 31099 & $2.26 \times 10^{3}$ & Regeneration & SOCS3, SOCS1 \\
\hline 46907 & $2.36 \times 10^{3}$ & Intracellular transport & KDELR1, GBF1, KIF1C, KIF1A \\
\hline
\end{tabular}


Table III. Continued.

$\mathrm{D}, \mathrm{M} 4$

\begin{tabular}{llll}
\hline GO-ID & P-value & \multicolumn{1}{c}{ Description } & \multicolumn{1}{c}{ Genes in test set } \\
\hline 16043 & $1.03 \times 10^{3}$ & Cellular component organization & TFRC, ARPC4, EPS15, SH3GL1 \\
16044 & $7.41 \times 10^{5}$ & Cellular membrane organization & TFRC, EPS15, SH3GL1 \\
61024 & $7.47 \times 10^{5}$ & Membrane organization & TFRC, EPS15, SH3GL1 \\
16192 & $2.71 \times 10^{4}$ & Vesicle-mediated transport & TFRC, EPS15, SH3GL1 \\
43623 & $6.55 \times 10^{4}$ & Cellular protein complex assembly & ARPC4, EPS15 \\
10324 & $1.41 \times 10^{3}$ & Membrane invagination & TFRC, SH3GL1 \\
6897 & $1.41 \times 10^{3}$ & Endocytosis & TFRC, SH3GL1 \\
34622 & $2.83 \times 10^{3}$ & Cellular macromolecular & ARPC4, EPS15 \\
34621 & $3.58 \times 10^{3}$ & Cellular macromolecular complex & ARPC4, EPS15 \\
6461 & $7.17 \times 10^{3}$ & Protein complex assembly & ARPC4, EPS15 \\
70271 & $7.17 \times 10^{3}$ & Protein complex biogenesis & ARPC4, EPS15 \\
65003 & $1.26 \times 10^{2}$ & Macromolecular complex assembly & ARPC4, EPS15 \\
\hline
\end{tabular}

E, M5

\begin{tabular}{|c|c|c|c|}
\hline GO-ID & P-value & Description & Genes in test set \\
\hline 6139 & $6.85 \times 10^{9}$ & $\begin{array}{l}\text { Nucleobase, nucleoside, nucleotide } \\
\text { and nucleic acid metabolic process }\end{array}$ & $\begin{array}{l}\text { NT5E, PABPN1, ENTPD4, SRSF1, POLR2D, AMPD1, } \\
\text { PPWD1, AKD1, CD2BP2, PRPF40A, CHERP }\end{array}$ \\
\hline 34641 & $3.49 \times 10^{8}$ & $\begin{array}{l}\text { Cellular nitrogen compound } \\
\text { metabolic process }\end{array}$ & $\begin{array}{l}\text { NT5E, PABPN1, ENTPD4, SRSF1, POLR2D, AMPD1, } \\
\text { PPWD1, AKD1, CD2BP2, PRPF40A, CHERP }\end{array}$ \\
\hline 6807 & $6.30 \times 10^{8}$ & $\begin{array}{l}\text { Nitrogen compound metabolic } \\
\text { process }\end{array}$ & $\begin{array}{l}\text { NT5E, PABPN1, ENTPD4, SRSF1, POLR2D, AMPD1, } \\
\text { PPWD1, AKD1, CD2BP2, PRPF40A, CHERP }\end{array}$ \\
\hline 44237 & $3.34 \times 10^{4}$ & Cellular metabolic process & $\begin{array}{l}\text { NT5E, PABPN1, ENTPD4, SRSF1, POLR2D, AMPD1, } \\
\text { PPWD1, AKD1, CD2BP2, PRPF40A, CHERP }\end{array}$ \\
\hline 44238 & $5.98 \times 10^{4}$ & Primary metabolic process & $\begin{array}{l}\text { NT5E, PABPN1, ENTPD4, SRSF1, POLR2D, AMPD1, } \\
\text { PPWD1, AKD1, CD2BP2, PRPF40A, CHERP }\end{array}$ \\
\hline 8152 & $1.95 \times 10^{3}$ & Metabolic process & $\begin{array}{l}\text { NT5E, PABPN1, ENTPD4, SRSF1, POLR2D, AMPD1, } \\
\text { PPWD1, AKD1, CD2BP2, PRPF40A, CHERP }\end{array}$ \\
\hline 90304 & $9.29 \times 10^{6}$ & Nucleic acid metabolic process & $\begin{array}{l}\text { NT5E, PABPN1, SRSF1, POLR2D, PPWD1, CD2BP2, } \\
\text { PRPF40A, CHERP }\end{array}$ \\
\hline 44260 & $4.95 \times 10^{3}$ & $\begin{array}{l}\text { Cellular macromolecule } \\
\text { metabolic process }\end{array}$ & $\begin{array}{l}\text { NT5E, PABPN1, SRSF1, POLR2D, PPWD1, CD2BP2, } \\
\text { PRPF40A, CHERP }\end{array}$ \\
\hline 43170 & $1.20 \times 10^{2}$ & Macromolecule metabolic process & $\begin{array}{l}\text { NT5E, PABPN1, SRSF1, POLR2D, PPWD1, CD2BP2, } \\
\text { PRPF40A, CHERP }\end{array}$ \\
\hline 6396 & $2.09 \times 10^{7}$ & RNA processing & $\begin{array}{l}\text { PABPN1, SRSF1, POLR2D, PPWD1, CD2BP2, PRPF40A, } \\
\text { CHERP }\end{array}$ \\
\hline 16070 & $7.49 \times 10^{6}$ & RNA metabolic process & $\begin{array}{l}\text { PABPN1, SRSF1, POLR2D, PPWD1, CD2BP2, PRPF40A, } \\
\text { CHERP }\end{array}$ \\
\hline 10467 & $4.36 \times 10^{5}$ & Gene expression & $\begin{array}{l}\text { PABPN1, SRSF1, POLR2D, PPWD1, CD2BP2, PRPF40A, } \\
\text { CHERP }\end{array}$ \\
\hline
\end{tabular}

F, M6

\begin{tabular}{llll}
\hline GO-ID & P-value & \multicolumn{1}{c}{ Description } & Genes in test set \\
\hline 48583 & $8.51 \times 10^{11}$ & Regulation of response to stimulus & IL10, CD40LG, SERPINE1, CCL2, AKT1, VEGFA, ICAM1 \\
32879 & $8.51 \times 10^{10}$ & Regulation of localization & IL10, CD40LG, SERPINE1, CCL2, AKT1, VEGFA, ICAM1 \\
48522 & $1.05 \times 10^{6}$ & Positive regulation of cellular process & IL10, CD40LG, SERPINE1, CCL2, AKT1, VEGFA, ICAM1
\end{tabular}


Table III. Continued.

\begin{tabular}{|c|c|c|c|}
\hline GO-ID & P-value & Description & Genes in test set \\
\hline 48518 & $2.07 \times 10^{6}$ & $\begin{array}{l}\text { Positive regulation of biological } \\
\text { process }\end{array}$ & IL10, CD40LG, SERPINE1, CCL2, AKT1, VEGFA, ICAM1 \\
\hline 50896 & $6.78 \times 10^{5}$ & Response to stimulus & IL10, CD40LG, SERPINE1, CCL2, AKT1, VEGFA, ICAM1 \\
\hline 50794 & $2.94 \times 10^{3}$ & Regulation of cellular process & IL10, CD40LG, SERPINE1, CCL2, AKT1, VEGFA, ICAM1 \\
\hline 50789 & $4.23 \times 10^{3}$ & Regulation of biological process & IL10, CD40LG, SERPINE1, CCL2, AKT1, VEGFA, ICAM1 \\
\hline 65007 & $6.33 \times 10^{3}$ & Biological regulation & IL10, CD40LG, SERPINE1, CCL2, AKT1, VEGFA, ICAM1 \\
\hline 43066 & $2.17 \times 10^{9}$ & Negative regulation of apoptosis & IL10, CD40LG, SERPINE1, CCL2, AKT1, VEGFA \\
\hline 43069 & $2.35 \times 10^{9}$ & $\begin{array}{l}\text { Negative regulation of programmed } \\
\text { cell death }\end{array}$ & IL10, CD40LG, SERPINE1, CCL2, AKT1, VEGFA \\
\hline 60548 & $2.66 \times 10^{9}$ & Negative regulation of cell death & IL10, CD40LG, SERPINE1, CCL2, AKT1, VEGFA \\
\hline 2682 & $4.47 \times 10^{9}$ & $\begin{array}{l}\text { Regulation of immune system } \\
\text { process }\end{array}$ & IL10, CD40LG, SERPINE1, CCL2, VEGFA, ICAM1 \\
\hline 42981 & $2.92 \times 10^{7}$ & Regulation of apoptosis & IL10, CD40LG, SERPINE1, CCL2, AKT1, VEGFA \\
\hline 43067 & $3.08 \times 10^{7}$ & $\begin{array}{l}\text { Regulation of programmed cell } \\
\text { death }\end{array}$ & IL10, CD40LG, SERPINE1, CCL2, AKT1, VEGFA \\
\hline 10941 & $3.24 \times 10^{7}$ & Regulation of cell death & IL10, CD40LG, SERPINE1, CCL2, AKT1, VEGFA \\
\hline 2376 & $5.51 \times 10^{7}$ & Immune system process & IL10, CD40LG, CCL2, AKT1, VEGFA, ICAM1 \\
\hline
\end{tabular}

G, M7

\begin{tabular}{|c|c|c|c|}
\hline GO-ID & P-value & Description & Genes in test set \\
\hline 23033 & $9.95 \times 10^{6}$ & Signaling pathway & TRIO, GRP, PTGER1, GNG4, KISS1R, PIK3R3 \\
\hline 7166 & $3.16 \times 10^{5}$ & $\begin{array}{l}\text { Cell surface receptor linked } \\
\text { signaling pathway }\end{array}$ & TRIO, GRP, PTGER1, KISS1R, PIK3R3 \\
\hline 23052 & $1.10 \times 10^{4}$ & Signaling & TRIO, GRP, PTGER1, GNG4, KISS1R, PIK3R3 \\
\hline 7186 & $7.80 \times 10^{4}$ & $\begin{array}{l}\text { G-protein coupled receptor protein } \\
\text { signaling pathway }\end{array}$ & GRP, PTGER1, KISS1R \\
\hline
\end{tabular}

GO, gene ontology; M, module.

eight of the hub genes were included in module 1 (COL2A1 and COL13A1; Fig. 2A), module 3 (SOCS3; Fig. 2C), module 6 (IL10, AKT1, ICAM1 and VEGFA; Fig. 2F) and module 7 (GNG4; Fig. 2G), suggesting that these eight genes may be particularly crucial for OLF.

Subsequently, BINGO was used to predict the function of these genes in the sub-modules. The results demonstrated that COL2A1 and COL13A1 in module 1 were involved in 'anatomical structure development', 'skeletal system development' and 'cell adhesion'; SOCS3 in module 3 was involved in 'negative regulation of insulin receptor signaling pathway', 'JAK-STAT cascade' and 'regeneration'; IL10, AKT1, ICAM1 and VEGFA in module 6 were involved in 'negative regulation of apoptosis' or 'regulation of immune system process'; and GNG4 in module 7 participated in 'signaling pathway' (Table III).

miRNA-mRNA regulatory network construction. A total of 876 negative miRNA-mRNA regulatory pairs (including miR-210-3p-IL10, hsa-miR-196a-5p-SOCS3, hsa-miR-379-5p-GNG4, has-miR-181b-5p-ADCY 5 , hsa-miR-329-3p-COL13A1, hsa-miR-222-5p-COL2A1 and hsa-miR-299-3p-WNT7B) were screened from the miRwalk database, which were used to construct a DEM-DEG network (Fig. 3). This constructed DEM-DEG regulatory network included 344 nodes, comprising of 73 DEMs (23 upregulated; 50 downregulated) and 271 DEGs (122 upregulated; 149 downregulated). GO biological process terms and KEGG pathways were analyzed to predict the potential functions of the DEGs in this DEM-DEG regulatory network using the DAVID database. The results demonstrated that these DEGs were enriched in $28 \mathrm{GO}$ biological processes, including 'GO:0042127 regulation of cell proliferation' (IL10 and VEGFA), 'GO:0002250 adaptive immune response' (IL10 and VEGFA), 'GO:0001501 skeletal system development' (COL2A1 and COL13A1), 'GO:0001503 ossification' (COL2A1 and COL13A1), 'GO:0060348 bone development' (COL2A1 and COL13A1), 'GO:0009725 response to hormone stimulus' (GNG4 and ADCY5), 'GO:0001666 response to hypoxia' (SOCS3) and 'GO:0042981 regulation of apoptosis' (SOCS3; Table IV; Fig. 4). In addition, these DEGs were enriched in eight KEGG pathways, including 'Hsa04510: Focal adhesion' (COL2A1 and VEGFA), 'Hsa00230: Purine metabolism' (ADCY5), 'Hsa04150: mTOR signaling pathway' (VEGFA), 'Hsa04310: Wnt signaling pathway' (WNT7B), 'Hsa04920: Adipocytokine signaling pathway' (SOCS3) 


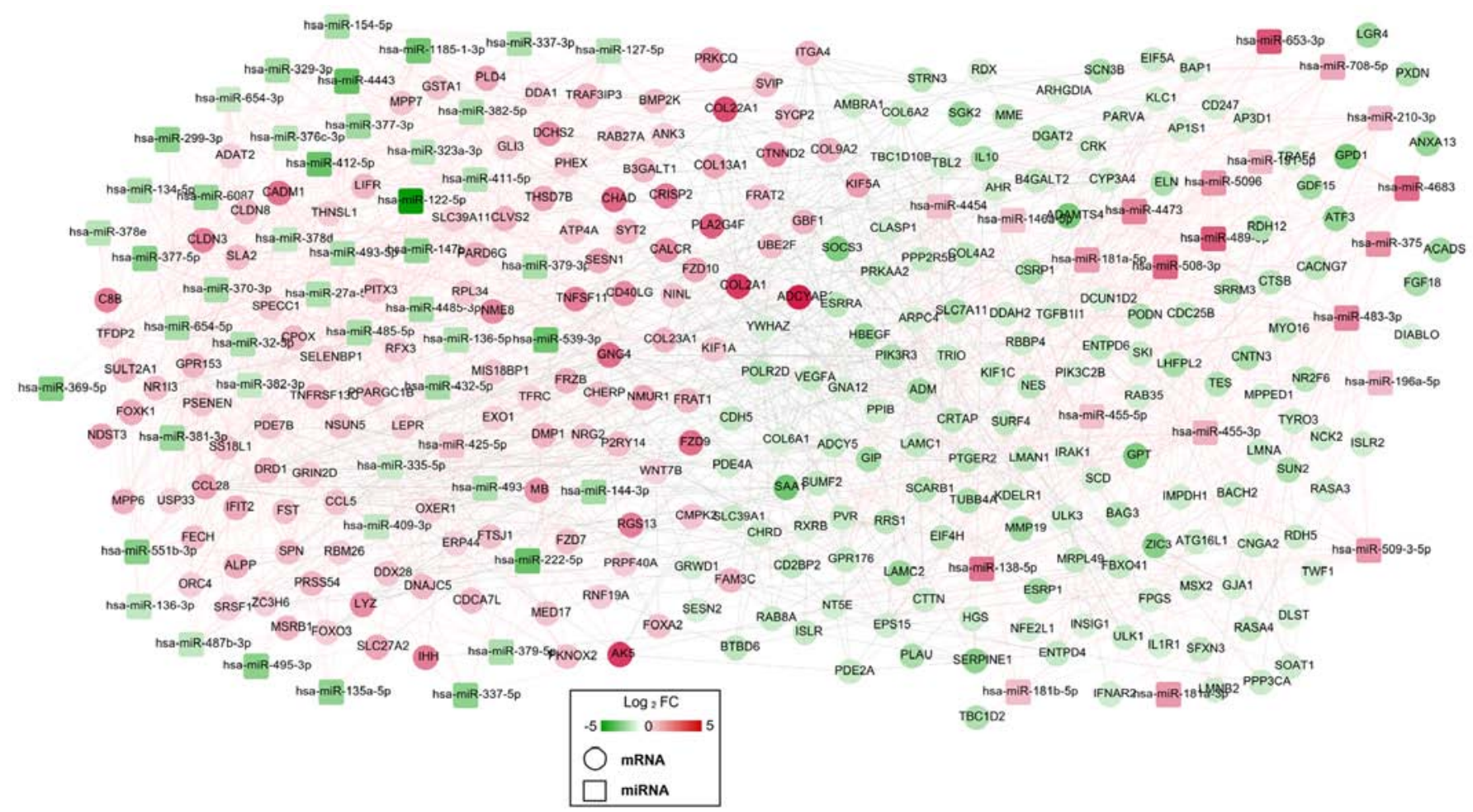

Figure 3. miRNA-mRNA interaction network. Red and green denoted upregulated and downregulated expression, respectively. The color depth is in proportion to differential levels. Squares represent miRNAs; circles represent mRNAs. mi, micro; miR, microRNA; FC, fold change.

and 'Hsa04660: T cell receptor signaling pathway' (IL10; Table IV; Fig. 4).

lncRNA-miRNA-mRNA ceRNA regulatory network construction. A total of 33 miRNA-lncRNA regulatory pairs [including small nucleolar RNA host gene (SNHG) 16-hsa-miR-196a-5p, ankyrin repeat and SOCS box containing 16 (ASB16)-AS1-hsa-miR-379-5p, nuclear enriched abundant transcript (NEAT) 1-has-miR-181b-5p and rhophilin (RHPN) 1-AS1-hsa-miR-299-3p] were screened from the starBase database, which were used to construct a DEM-DEL regulatory network (Fig. 5). This established DEM-DEL regulatory network included 31 nodes, comprising of 22 DEMs (9 upregulated; 13 downregulated) and nine DELs (6 upregulated; 3 downregulated).

Following the integration of the DEM-DEG and DEM-DEL regulatory networks, an IncRNA-miRNA-mRNA ceRNA network (including SNHG16-hsa-miR-196a-5p-SOCS3, ASB16-AS1-hsa-miR-379-5p-GNG4, NEAT1-has-miR181b-5p-ADCY5 and RHPN1-AS1-hsa-miR-299-3p-WNT7B) was constructed (Fig. 6), in which 165 nodes (8 DELs; 21 DEMs; 136 DEGs) and 245 edges (32 DEL-DEM and 213 DEM-DEG interaction pairs) were involved. The functional analysis of the genes in this ceRNA network also demonstrated that 'GO:0009725 response to hormone stimulus' (GNG4 and ADCY5), 'GO:0001666 response to hypoxia' (SOCS3), 'Hsa00230: Purine metabolism' (ADCY5), 'Hsa04920: Adipocytokine signaling pathway' (SOCS3), 'Hsa04062: Chemokine signaling pathway' (GNG4 and ADCY5) and 'Hsa04310: Wnt signaling pathway' (WNT7B) were enriched (Table V; Fig. 7).

\section{Discussion}

Although the same datasets were used from the study by Han et al (22), the present study applied several different bioinformatics methods aiming to screen crucial molecular mechanisms for OLF: i) Hub genes were identified by constructing the PPI network, ranking the nodes according to the topological properties and extracting the sub-modules; ii) the target genes of miRNAs were predicted using the miRwalk database, which contained 12 prediction algorithms, not only three; and iii) the key lncRNAs were identified on the basis of the IncRNA-miRNA-mRNA ceRNA regulatory network, not the lncRNA-mRNA co-expression network. Accordingly, the present study may provide certain novel miRNAs and lncRNAs for explaining the pathogenesis of OLF, and developing novel therapeutic approaches for OLF. As a result, it was identified, for the first time to the best of the authors' knowledge, that miR-210-3p may be a key miRNA for OLF by regulating immune-related gene IL10. lncRNA SNHG16, ASB16-AS1 and NEAT1 may also be important by acting as ceRNAs for miR-196a-5p, miR-379-5p and miR-181b-5p to modulate the expression levels of miRNA target genes SOCS3, GNG4 and ADCY5, respectively. SOCS3 was involved in 'response to hypoxia', 'regulation of apoptosis' and 'regeneration', while GNG4 and ADCY5 participated in the 'Chemokine signaling pathway'. All these mRNAs were hub genes in the PPI network.

Previous studies have demonstrated that inflammatory cytokines promote hypertrophy and ossification of LF cells, but only a number of them (TNF- $\alpha$, IL-1 $\alpha$ and IL-6) have been investigated (13-15). The present study predicted that IL10, 
Table IV. Function enrichment for genes in microRNA-mRNA network.

A, Biological process

\begin{tabular}{lll}
\hline Term & P-value & Genes \\
\hline
\end{tabular}

GO:0042127 regulation of cell proliferation

GO:0002250 adaptive immune response

GO:0010033 response to organic substance

$2.87 \times 10^{4}$

$4.04 \times 10^{4}$

GO:0001501 skeletal system development

$9.07 \times 10^{4}$

GO:0009719 response to endogenous stimulus

$1.23 \times 10^{3}$

GO:0009725 response to hormone stimulus

$1.24 \times 10^{3}$

GO:0042060 wound healing

$1.36 \times 10^{3}$

GO:0009611 response to wounding

$1.37 \times 10^{3}$

GO:0007155 cell adhesion

$1.42 \times 10^{3}$

GO:0022610 biological adhesion

$1.45 \times 10^{3}$

GO:0002684 positive regulation of immune system process

GO:0007599 hemostasis

GO:0016337 cell-cell adhesion

GO:0008285 negative regulation of

cell proliferation

GO:0048545 response to steroid

hormone stimulus

GO:0016477 cell migration

GO:0016055 Wnt receptor signaling pathway
$2.14 \times 10^{3}$

$2.16 \times 10^{3}$

$2.28 \times 10^{3}$

$2.91 \times 10^{3}$

$4.86 \times 10^{3}$

$6.55 \times 10^{3}$

$6.81 \times 10^{3}$
FGF18, BAP1, EIF5A, GJA1, SESN1, GLI3, IL10, CDH5, MSX2, SERPINE1, SPN, IHH, PTGER2, ESRRA, RBBP4, TNFRSF13C, LIFR, SKI, CDC25B, NCK2, PRKCQ, ATF3, ADM, VEGFA, MYO16, HBEGF, HGS, LAMC1, TGFB1I1, PLAU

EXO1, C8B, CADM1, CD40LG, SLA2, VEGFA, IL10, RAB27A

CALCR, IL1R1, DRD1, LEPR, ADCY5, CCL5, IL10, MSX2, COL6A2, SCARB1, PPP3CA, PIK3R3, GNG4, DDAH2, IHH, MB, IRAK1, ACADS, SOCS3, STRN3, LIFR, PPARGC1B, ERP44, PRKCQ, ADM, TFRC FGF18, ESRRA, COL13A1, DMP1, COL2A1, FRZB, GLI3, CHAD, MSX2, COL9A2, TNFSF11, PHEX, CHRD, ADAMTS4, IHH

CALCR, DRD1, ACADS, SOCS3, STRN3, LEPR, ADCY5, CCL5, IL10, PPARGC1B, PRKCQ, ADM, PPP3CA, PIK3R3, GNG4, IHH, MB

CALCR, DRD1, ACADS, SOCS3, STRN3, LEPR, ADCY5, CCL5, IL10, PPARGC1B, PRKCQ, ADM, PIK3R3, GNG4, IHH, MB

PRKCQ, FOXA2, CD40LG, SAA1, SERPINE1, GNA12, HBEGF, SCARB1, LMAN1, PLAU, RAB27A YWHAZ, FOXA2, GNA12, LYZ, LMAN1, CCL5, IL10, C8B, PRKCQ, ADM, TFRC, CD40LG, SAA1, SERPINE1, HBEGF, NFE2L1, SCARB1, CTSB, PLAU, RAB27A

PVR, CLDN8, DCHS2, TYRO3, CADM1, COL13A1, CLDN3, COL22A1, CTNND2, COL2A1, ITGA4, CCL5, CDH5, CHAD, ISLR, CD40LG, COL6A2, COL6A1, LAMC2, SCARB1, CNTN3, LAMC1, TGFB1I1, PARVA

PVR, CLDN8, DCHS2, TYRO3, CADM1, COL13A1, CLDN3, COL22A1, CTNND2, COL2A1, ITGA4, CCL5, CDH5, CHAD, ISLR, CD40LG, COL6A2, COL6A1, LAMC2, SCARB1, CNTN3, LAMC1, TGFB1I1, PARVA PVR, C8B, IRAK1, PRKCQ, NCK2, CADM1, SLA2, VEGFA, CD247, TNFRSF13C, AP3D1, SPN FOXA2, CD40LG, SAA1, SERPINE1, GNA12, LMAN1, PLAU, RAB27A

PVR, CLDN8, DCHS2, CADM1, COL13A1, CD40LG, CLDN3, CTNND2, COL6A2, COL2A1, ITGA4, CDH5, CHAD

RBBP4, BAP1, GJA1, SKI, SESN1, GLI3, IL10, CDH5, MSX2, NCK2, ADM, MYO16, HGS, TGFB1I1, SPN CALCR, ADM, SOCS3, ACADS, STRN3, LEPR, CCL5, IL10, PPARGC1B, IHH PVR, NCK2, DRD1, ULK1, SAA1, HBEGF, SCARB1, LAMC1, ITGA4, CCL5, IL10, PLAU FZD9, WNT7B, FZD10, FRAT1, FRAT2, TGFB1I1, FRZB, FZD7 
Table IV. Continued.

\begin{tabular}{|c|c|c|}
\hline Term & P-value & Genes \\
\hline GO:0001666 response to hypoxia & $7.08 \times 10^{3}$ & $\begin{array}{l}\text { PRKCQ, TFRC, ADM, SOCS3, CLDN3, VEGFA, } \\
\text { PLAU, MB }\end{array}$ \\
\hline GO:0042981 regulation of apoptosis & $7.81 \times 10^{3}$ & $\begin{array}{l}\text { IRAK1, YWHAZ, CADM1, SOCS3, EIF5A, TRIO, } \\
\text { COL2A1, FOXO3, GLI3, IL10, MSX2, CD40LG, } \\
\text { BAG3, VEGFA, DIABLO, PSENEN, DNAJC5, CTSB, } \\
\text { DDAH2, ARHGDIA, TRAF4, SPN, RAB27A, IHH }\end{array}$ \\
\hline $\begin{array}{l}\text { GO:0044271 nitrogen compound biosynthetic } \\
\text { process }\end{array}$ & $8.29 \times 10^{3}$ & $\begin{array}{l}\text { FECH, ATP4A, ADCY5, AK5, CMPK2, ADM, CPOX, } \\
\text { NFE2L1, THNSL1, FPGS, DDAH2, IMPDH1, NT5E }\end{array}$ \\
\hline $\begin{array}{l}\text { GO:0010648 negative regulation of cell } \\
\text { communication }\end{array}$ & $8.62 \times 10^{3}$ & $\begin{array}{l}\text { DRD1, SOCS3, STRN3, SLA2, HGS, SKI, TGFB1I1, } \\
\text { FRZB, CHRD, IHH, RGS13 }\end{array}$ \\
\hline $\begin{array}{l}\text { GO:0043067 regulation of programmed } \\
\text { cell death }\end{array}$ & $8.74 \times 10^{3}$ & $\begin{array}{l}\text { IRAK1, YWHAZ, CADM1, SOCS3, EIF5A, TRIO, } \\
\text { COL2A1, FOXO3, GLI3, IL10, MSX2, CD40LG, } \\
\text { BAG3, VEGFA, DIABLO, PSENEN, DNAJC5, CTSB, } \\
\text { DDAH2, ARHGDIA, TRAF4, SPN, RAB27A, IHH }\end{array}$ \\
\hline GO:0010941 regulation of cell death & $9.12 \times 10^{3}$ & $\begin{array}{l}\text { IRAK1, YWHAZ, CADM1, SOCS3, EIF5A, TRIO, } \\
\text { COL2A1, FOXO3, GLI3, IL10, MSX2, CD40LG, } \\
\text { BAG3, VEGFA, DIABLO, PSENEN, DNAJC5, CTSB, } \\
\text { DDAH2, ARHGDIA, TRAF4, SPN, RAB27A, IHH }\end{array}$ \\
\hline GO:0001503 ossification & $1.24 \times 10^{2}$ & $\begin{array}{l}\text { FGF18, TNFSF11, COL13A1, DMP1, COL2A1, CHRD, } \\
\text { IHH }\end{array}$ \\
\hline GO:0048870 cell motility & $1.38 \times 10^{2}$ & $\begin{array}{l}\text { PVR, NCK2, DRD1, ULK1, SAA1, HBEGF, SCARB1, } \\
\text { LAMC1, ITGA4, CCL5, IL10, PLAU }\end{array}$ \\
\hline GO:0051674 localization of cell & $1.38 \times 10^{2}$ & $\begin{array}{l}\text { PVR, NCK2, DRD1, ULK1, SAA1, HBEGF, SCARB1, } \\
\text { LAMC1, ITGA4, CCL5, IL10, PLAU }\end{array}$ \\
\hline GO:0043066 negative regulation of apoptosis & $1.55 \times 10^{2}$ & $\begin{array}{l}\text { MSX2, IRAK1, YWHAZ, CD40LG, SOCS3, BAG3, } \\
\text { VEGFA, DNAJC5, COL2A1, DDAH2, IL10, } \\
\text { ARHGDIA, IHH }\end{array}$ \\
\hline GO:0060348 bone development & $1.68 \times 10^{2}$ & $\begin{array}{l}\text { FGF18, TNFSF11, COL13A1, DMP1, COL2A1, CHRD, } \\
\text { IHH }\end{array}$ \\
\hline
\end{tabular}

B, KEGG pathway

\begin{tabular}{|c|c|c|}
\hline Term & P-value & Genes \\
\hline hsa04512:ECM-receptor interaction & $4.33 \times 10^{3}$ & $\begin{array}{l}\text { COL4A2, COL6A2, COL6A1, LAMC2, COL2A1, } \\
\text { LAMC1, ITGA4, CHAD }\end{array}$ \\
\hline hsa04510:Focal adhesion & $1.00 \times 10^{2}$ & $\begin{array}{l}\text { COL4A2, VEGFA, COL6A2, COL6A1, LAMC2, } \\
\text { COL2A1, LAMC1, ITGA4, PIK3R3, CRK, CHAD, } \\
\text { PARVA }\end{array}$ \\
\hline hsa00230:Purine metabolism & $1.25 \times 10^{2}$ & $\begin{array}{l}\text { PDE7B, PDE2A, ADCY5, PDE4A, ENTPD6, AK5, } \\
\text { ENTPD4, POLR2D, NT5E, IMPDH1 }\end{array}$ \\
\hline hsa04150:mTOR signaling pathway & $3.80 \times 10^{2}$ & ULK1, VEGFA, ULK3, PRKAA2, PIK3R3 \\
\hline hsa04514:Cell adhesion molecules (CAMs) & $4.30 \times 10^{2}$ & $\begin{array}{l}\text { PVR, CLDN8, CADM1, CD40LG, CLDN3, ITGA4, } \\
\text { CDH5, SPN }\end{array}$ \\
\hline hsa04310:Wnt signaling pathway & $4.77 \times 10^{2}$ & $\begin{array}{l}\text { FZD9, WNT7B, FZD10, PPP2R5B, FRAT1, FRAT2, } \\
\text { PPP3CA, FZD7 }\end{array}$ \\
\hline hsa04920:Adipocytokine signaling pathway & $4.82 \times 10^{2}$ & PRKCQ, SOCS3, RXRB, LEPR, PRKAA2 \\
\hline hsa04660:T cell receptor signaling pathway & $4.90 \times 10^{2}$ & $\begin{array}{l}\text { PRKCQ, NCK2, CD40LG, CD247, PPP3CA, PIK3R3, } \\
\text { IL10 }\end{array}$ \\
\hline
\end{tabular}




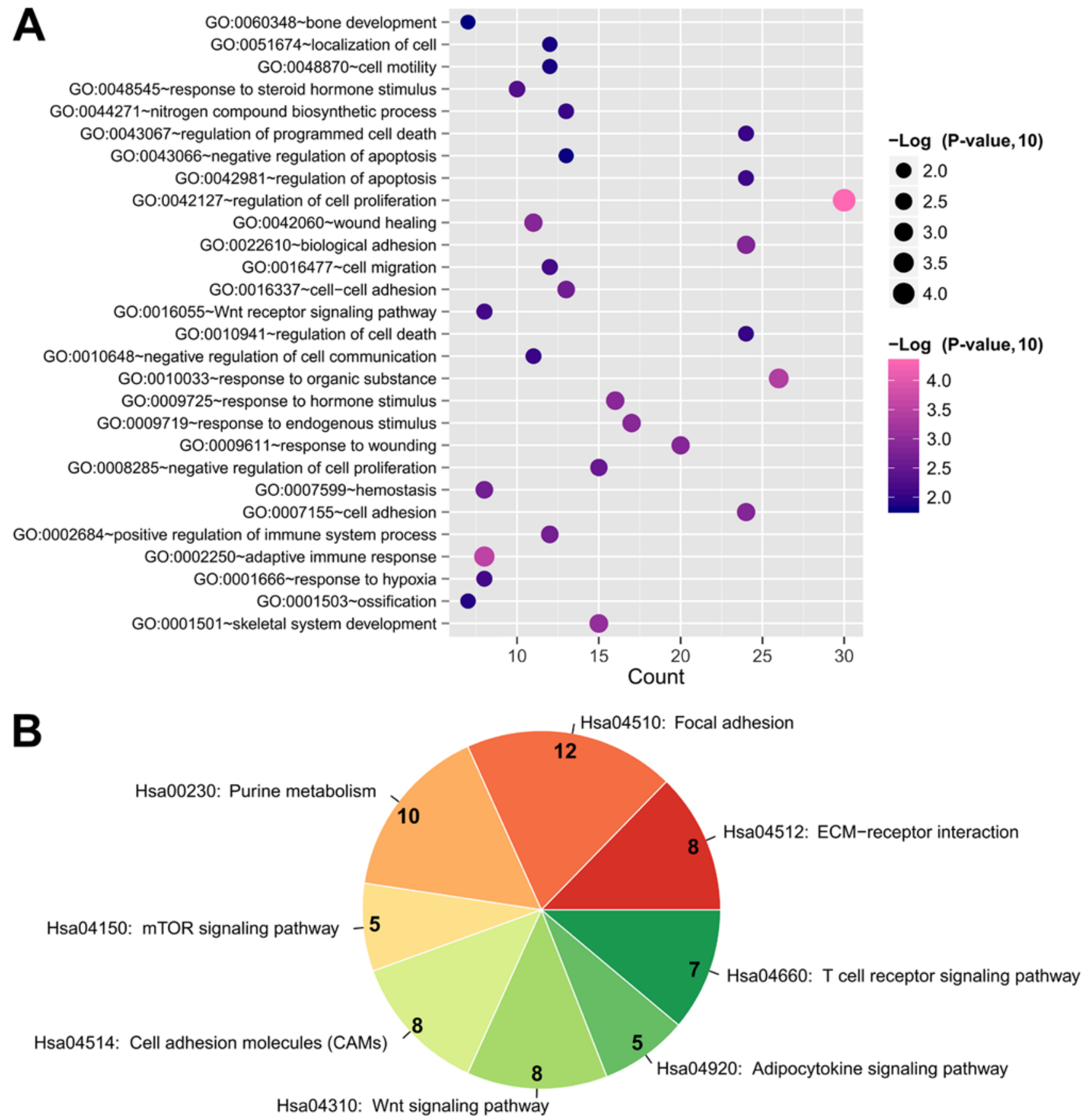

Figure 4. Function enrichment for the genes in the microRNA-mRNA interaction network. (A) GO analysis and (B) KEGG pathways. GO, Gene Ontology; KEGG, Kyoto Encyclopedia of Genes and Genomes; ECM, extracellular matrix.

SOCS3 and ADCY5 may be anti-inflammatory due to their downregulation, while GNG4 may be pro-inflammatory due to its upregulation in OLF. The associations of the identified genes with inflammation can be indirectly confirmed. For example, IL10 is a known anti-inflammatory cytokine that was identified to have lower expression in subligamentous type of disc degeneration (8). SOCS3 may mediate the blockade of inflammation by inhibiting Janus kinase-STAT3 activity and to prevent the abnormal expression of IL-6 $(32,33)$. ADCY5 was also demonstrated to be significantly downregulated in cytokine-related hepatocellular carcinoma (34) and prostate cancer (35). Although GNG4 was previously demonstrated to be downregulated in glioblastoma cells and exogenous overexpression of GNG4 can inhibit stromal cell-derived factor 1/C-X-C motif chemokine receptor 4-dependent chemokine signaling (36), two recent studies observed that GNG4 was significantly upregulated in patients with colon cancer (37) and cardiovascular events (38), indicating its potential pro-inflammatory and pro-proliferation roles. In agreement with these two studies, the present study additionally identified that GNG4 was upregulated in LF cells.

Although there have been previous studies that examined the roles of miRNAs in OLF, all of these studies focused on miRNAs that regulate osteogenic differentiation related genes (17-20,39). miRNAs related with inflammation and cell proliferation in OLF have rarely been reported. Using 


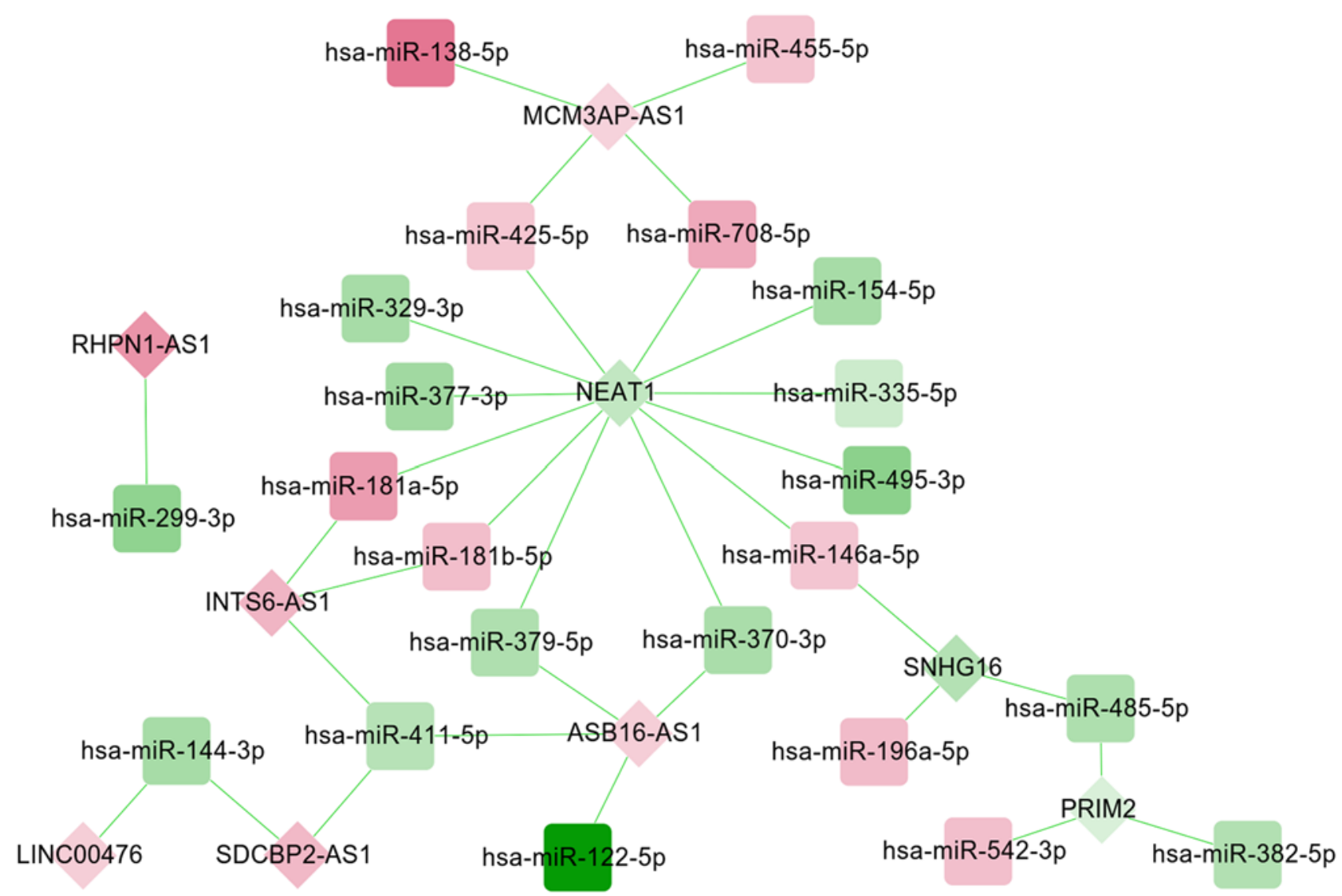

Figure 5. IncRNA-miRNA interaction network. Diamonds represent lncRNAs and squares represent miRNAs. Red and green denoted upregulated and downregulated expression, respectively. The color depth is in proportion to differential levels. lnc, long non-coding RNA; miRNA/miR, microRNA.

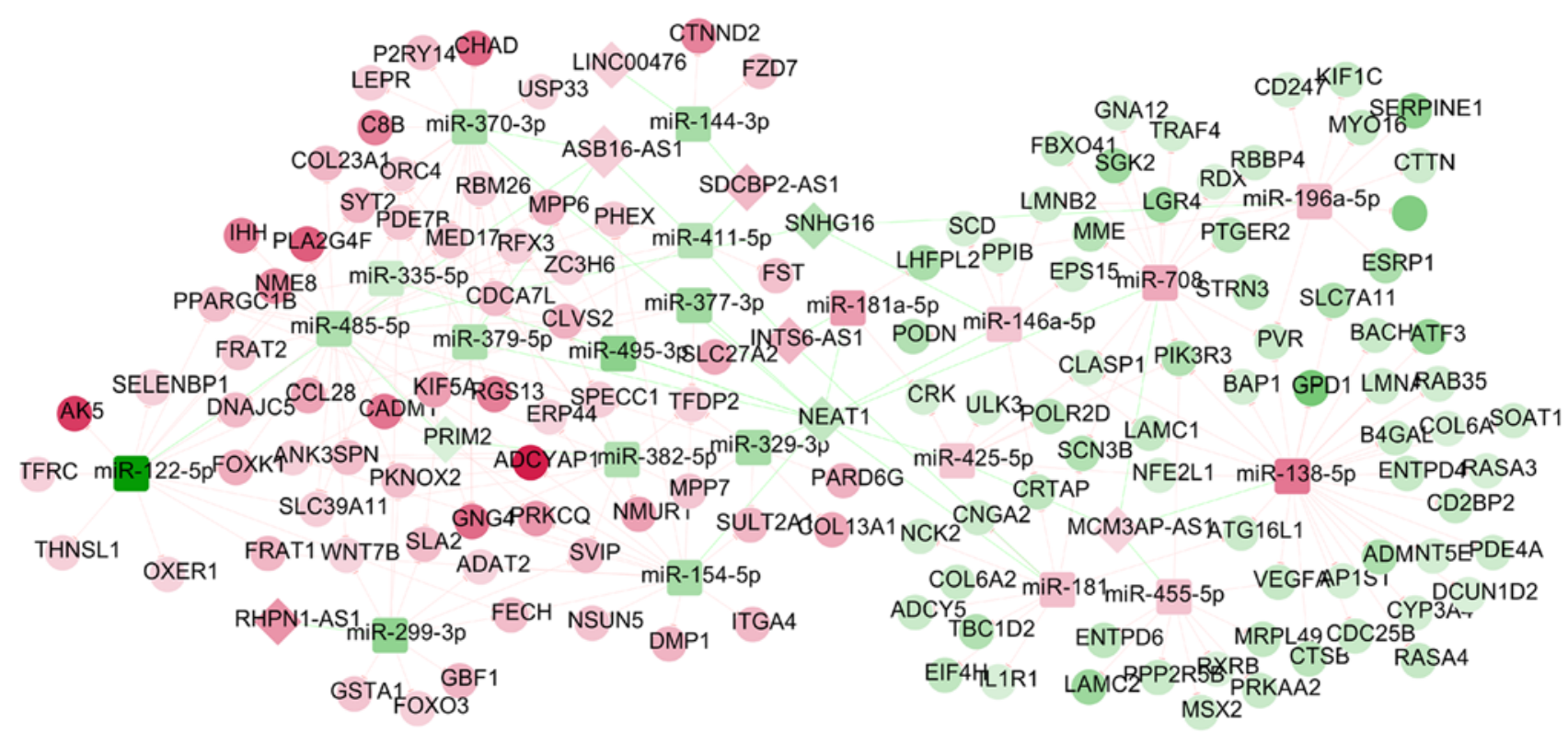

Figure 6. Competing endogenous RNA interaction network of lncRNA-miRNA-mRNA. Red and green denoted upregulated and downregulated expression, respectively. The color depth is in proportion to differential levels. Squares represent miRNAs; circles represent mRNAs; and diamonds represent lncRNAs. lncRNA, long non-coding RNA; miRNA/miR, microRNA.

comprehensive analysis, the present study identified that miR-210-3p, miR-196a-5p and miR-181b-5p targeting anti-inflammatory IL10, SOCS3 and ADCY5, respectively, were upregulated, but miR-379-5p, which targets pro-inflammatory GNG4, was downregulated in OLF. The interaction associations between miR-210 and miR-196 and their target genes has been demonstrated in other inflammatory diseases. For example, administration of agomir-210 
Table V. Function enrichment for genes in the long non-coding RNA-microRNA-mRNA competing endogenous RNA network.

A, Biological process

\begin{tabular}{|c|c|c|}
\hline Term & P-value & Genes \\
\hline $\begin{array}{l}\text { GO:0002684 positive regulation of immune } \\
\text { system process }\end{array}$ & $1.01 \times 10^{3}$ & $\begin{array}{l}\text { PVR, C8B, PRKCQ, NCK2, CADM1, SLA2, VEGFA, } \\
\text { CD247, SPN }\end{array}$ \\
\hline GO:0010033 response to organic substance & $3.44 \times 10^{3}$ & $\begin{array}{l}\text { IL1R1, SOCS3, STRN3, LEPR, ADCY5, PPARGC1B, } \\
\text { MSX2, ERP44, PRKCQ, ADM, TFRC, COL6A2, } \\
\text { PIK3R3, GNG4, IHH }\end{array}$ \\
\hline $\begin{array}{l}\text { GO:0007166 cell surface receptor linked } \\
\text { signal transduction }\end{array}$ & $3.57 \times 10^{3}$ & $\begin{array}{l}\text { IL1R1, LEPR, ADCY5, GNA12, CD247, FST, CCL28, } \\
\text { LGR4, ADCYAP1, MSX2, NMUR1, OXER1, FRAT1, } \\
\text { FRAT2, GNG4, PIK3R3, SPN,PTGER2, SLA2, } \\
\text { ITGA4, FZD7, RGS13, EPS15, NCK2, WNT7B, } \\
\text { ADM, P2RY14, VEGFA }\end{array}$ \\
\hline GO:0009725 response to hormone stimulus & $4.30 \times 10^{3}$ & $\begin{array}{l}\text { PRKCQ, ADM, SOCS3, STRN3, ADCY5, LEPR, } \\
\text { PIK3R3, GNG4, PPARGC1B, IHH }\end{array}$ \\
\hline GO:0042127 regulation of cell proliferation & $7.37 \times 10^{3}$ & $\begin{array}{l}\text { PTGER2, RBBP4, BAP1, CDC25B, MSX2, PRKCQ, } \\
\text { NCK2, ATF3, ADM, VEGFA, SERPINE1, MYO16, } \\
\text { LAMC1, SPN, IHH }\end{array}$ \\
\hline GO:0009719 response to endogenous stimulus & $8.05 \times 10^{3}$ & $\begin{array}{l}\text { PRKCQ, ADM, SOCS3, STRN3, ADCY5, LEPR, } \\
\text { PIK3R3, GNG4, PPARGC1B, IHH }\end{array}$ \\
\hline GO:0043062 extracellular structure organization & $1.31 \times 10^{2}$ & WNT7B, CADM1, ANK3, DMP1, COL6A2, LAMC1 \\
\hline GO:0007568 aging & $1.48 \times 10^{2}$ & PRKCQ, TFRC, ADM, SOCS3, SERPINE1 \\
\hline $\begin{array}{l}\text { GO:0045137 development of primary sexual } \\
\text { characteristics }\end{array}$ & $2.37 \times 10^{2}$ & LEPR, FST, VEGFA, FOXO3, LGR4 \\
\hline GO:0048545 response to steroid hormone stimulus & $2.48 \times 10^{2}$ & ADM, SOCS3, STRN3, LEPR, PPARGC1B, IHH \\
\hline GO:0008284 positive regulation of cell proliferation & $2.60 \times 10^{2}$ & $\begin{array}{l}\text { PRKCQ, NCK2, ATF3, ADM, VEGFA, LAMC1, } \\
\text { SPN, CDC25B, IHH }\end{array}$ \\
\hline GO:0001666 response to hypoxia & $2.81 \times 10^{2}$ & PRKCQ, TFRC, ADM, SOCS3, VEGFA \\
\hline GO:0008202 steroid metabolic process & $2.99 \times 10^{2}$ & CYP3A4, SOAT1, SULT2A1, ADM, LEPR, PRKAA2 \\
\hline GO:0016337 cell-cell adhesion & $3.15 \times 10^{2}$ & $\begin{array}{l}\text { PVR, CADM1, COL13A1, CTNND2, COL6A2, } \\
\text { ITGA4, CHAD }\end{array}$ \\
\hline GO:0070482 response to oxygen levels & $3.31 \times 10^{2}$ & PRKCQ, TFRC, ADM, SOCS3, VEGFA \\
\hline GO:0050778 positive regulation of immune response & $3.61 \times 10^{2}$ & PVR, C8B, CADM1, SLA2, CD247 \\
\hline GO:0007548 sex differentiation & $4.09 \times 10^{2}$ & LEPR, FST, VEGFA, FOXO3, LGR4 \\
\hline
\end{tabular}

B, KEGG pathway

\begin{tabular}{|c|c|c|}
\hline Term & P-value & Genes \\
\hline hsa00230:Purine metabolism & $3.14 \times 10^{3}$ & $\begin{array}{l}\text { PDE7B, ADCY5, PDE4A, ENTPD6, AK5, ENTPD4, } \\
\text { POLR2D, NT5E }\end{array}$ \\
\hline hsa04510:Focal adhesion & $3.77 \times 10^{3}$ & $\begin{array}{l}\text { VEGFA, COL6A2, COL6A1, LAMC2, LAMC1, } \\
\text { ITGA4, PIK3R3, CRK, CHAD }\end{array}$ \\
\hline hsa04512:ECM-receptor interaction & $4.15 \times 10^{3}$ & COL6A2, COL6A1, LAMC2, LAMC1, ITGA4, CHAD \\
\hline hsa04920:Adipocytokine signaling pathway & $1.02 \times 10^{2}$ & PRKCQ, SOCS3, RXRB, LEPR, PRKAA2 \\
\hline hsa04150:mTOR signaling pathway & $2.82 \times 10^{2}$ & VEGFA, ULK3, PRKAA2, PIK3R3 \\
\hline hsa04062:Chemokine signaling pathway & $2.90 \times 10^{2}$ & ADCY5, FOXO3, PIK3R3, GNG4, CRK, CCL28 \\
\hline hsa04310:Wnt signaling pathway & $3.13 \times 10^{2}$ & WNT7B, PPP2R5B, FRAT1, FRAT2, FZD7 \\
\hline hsa04660:T cell receptor signaling pathway & $3.16 \times 10^{2}$ & PRKCQ, NCK2, CD247, PIK3R3 \\
\hline hsa03320:PPAR signaling pathway & $3.22 \times 10^{2}$ & RXRB, SCD, SLC27A2 \\
\hline hsa04514:Cell adhesion molecules (CAMs) & $3.23 \times 10^{2}$ & PVR, CADM1, ITGA4, SPN \\
\hline hsa04810:Regulation of actin cytoskeleton & $3.29 \times 10^{2}$ & GNA12, RDX, ITGA4, PIK3R3, CRK \\
\hline
\end{tabular}




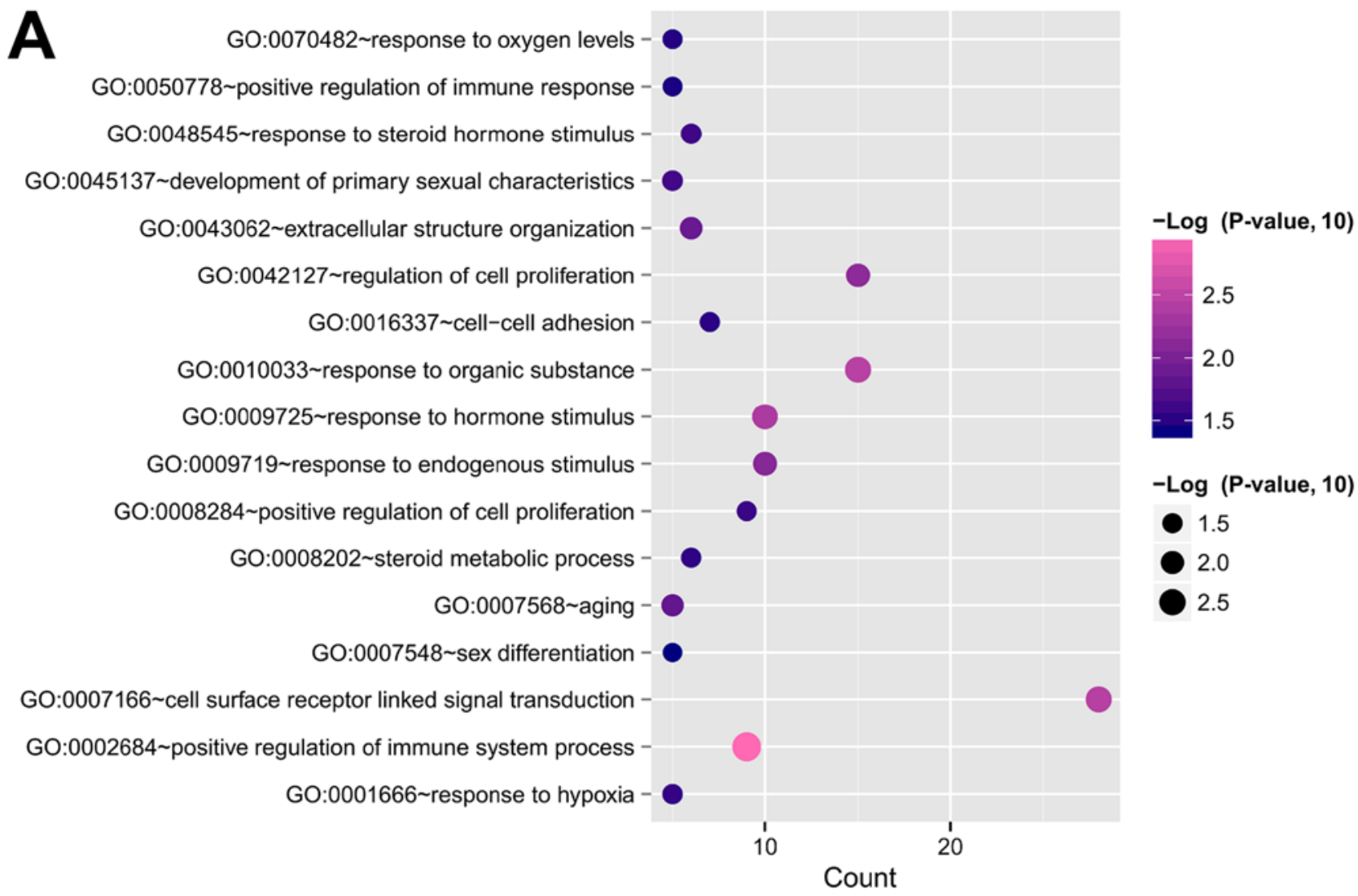

B

Hsa04510: Focal adhesion

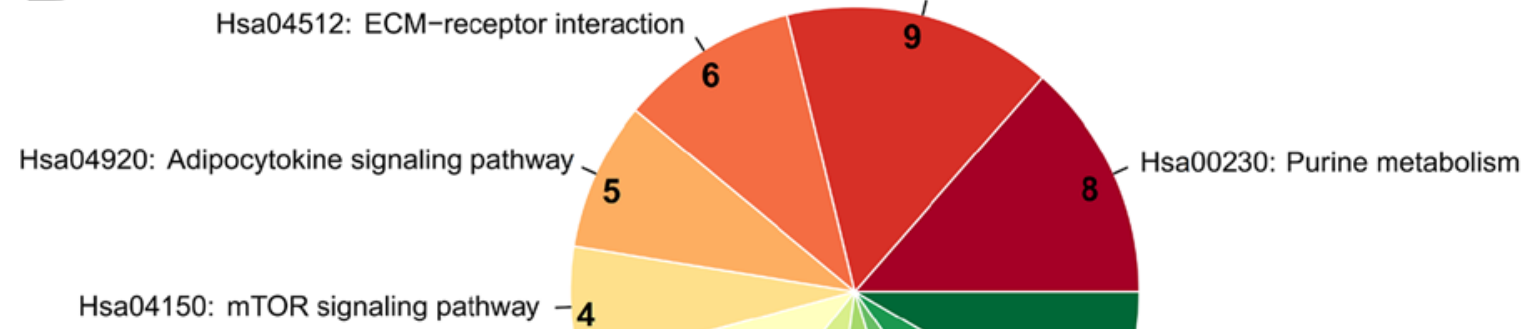

Hsa04150: mTOR signaling pathway -4

Hsa04062: Chemokine signaling pathway -6

Hsa04310: Wnt signaling pathway

5

4

3

Hsa04514: Cell adhesion molecules (CAMs)

Hsa04660: T cell receptor signaling pathway

Figure 7. Function enrichment for the genes in the lncRNA-miRNA-mRNA interaction network. (A) GO analysis and (B) KEGG pathways. lncRNA, long non-coding RNA; miRNA, microRNA; GO, Gene Ontology; KEGG, Kyoto Encyclopedia of Genes and Genomes.

significantly upregulated IL-10 and attenuated cellular apoptosis and inflammation in an injured rat spinal cord, ultimately improving functional recovery (40). Ectopic expression of miR-196 promoted stemness and chemoresistance of colorectal cancer cells by targeting SOCS3, a negative regulator of the STAT3 signaling pathway (41). miR-181b has been reported to stimulate inflammation via the nuclear factor- $\kappa \mathrm{B}$ signaling pathway (42), while miR-379 significantly suppresses the invasive capacity of cancer cells by inhibiting cytokine IL-18 (43). These findings may indirectly verify the important roles of these miRNAs in inflammatory OLF.
Furthermore, the present study also identified several crucial lncRNAs that regulated the mentioned inflammation and cell proliferation related genes based on the ceRNA hypothesis, including downregulated lncRNA SNHG16/NEAT1 and upregulated ASB16-AS1. Although their mechanisms in OLF require confirmation in further experiments, previous studies have indirectly identified their underlying associations. Zhao et al (44) demonstrated that NEAT1 was decreased in primary acute myeloid leukemia cells and THP-1 monocytes compared with normal cells; overexpression of NEAT1 inhibits cell proliferation, 
promotes apoptosis and affects the cell cycle. Overexpressed ASB16-AS1 has been reported to increase the expression of osteoblastogenesis-related genes (BMP2 and ALP) (45) which were previously demonstrated to be induced by inflammatory cytokines (14). The roles of SNHG16 on cell proliferation may be controversial, although the majority of studies have demonstrated that SNHG16 may functions as an oncogene $(46,47)$. However, the present study identified that expression of SNHG16 decreased in LF cells of patients with OLF compared with the controls and further investigation is necessary to elucidate the underlying biological associations between SNHG16 and OLF.

In addition to inflammation genes, the present study also identified the significant miRNAs and IncRNAs associated with osteogenic differentiation related genes. miR-329-3p and miR-222-5p were involved in ossification by regulating COL13A1 and COL2A1, respectively. RHPN1-AS1 functioned as a ceRNA for miR-299-3p to influence the Wnt signaling pathway through WNT7B. These results were in agreement with a previous study, in which inhibition of miR-222-3p in human bone mesenchymal stem cells promoted the expression of osteoblast-specific genes, ALP activity, and matrix mineralization, while overexpression of miR-222-3p inhibited osteoblast differentiation (48). The roles of other miRNAs and lncRNAs require further investigation.

There are certain limitations to the present study. Only two datasets were included to examine the molecular mechanisms of OLF due to limited previous studies. Also, the current sample size of these datasets was small. Therefore, further studies using high-throughput sequencing experiments with larger clinical samples would be valuable. Another limitation is that this is a preliminary study to identify the crucial miRNAs and lncRNAs for OLF. Further in vitro and in vivo experiments are necessary to confirm the expression levels of these identified miRNAs and lncRNAs in OLF, and to demonstrate the regulatory associations between them and the downstream DEGs.

In conclusion, the present study identified several inflammation and osteogenic differentiation related miRNA-mRNAs (miR-210-3p-IL10, hsa-miR-329-3p-COL13A1 and hsa-miR-222-5p-COL2A1) or lncRNA-miRNA-mRNA interaction axes (SNHG16-hsa-miR-196a-5p-SOCS3, ASB16-AS1-hsa-miR-379-5p-GNG4, NEAT1-has-miR-181b5p-ADCY5 and RHPN1-AS1-hsa-miR-299-3p-WNT7B), which may be involved in the pathogenesis of OLF. These miRNAs and lncRNAs may be natural, endogenous and nontoxic drug targets for the treatment of OLF.

\section{Acknowledgements}

Not applicable.

\section{Funding}

No funding was received.

\section{Availability of data and materials}

The microarray data GSE106253 and GSE106256 were downloaded from The Gene Expression Omnibus database in
National Center for Biotechnology Information (http://www. ncbi.nlm.nih.gov/geo/).

\section{Authors' contributions}

DK and FW were involved in the conception and design of this study. DK and QZ collected the data and performed the bioinformatics analyses. WL prepared the figures and interpreted the data. DK drafted the manuscript. FW revised the manuscript. All authors read and approved the final manuscript.

\section{Ethics approval and consent to participate}

Not applicable.

\section{Patient consent for publication}

Not applicable.

\section{Competing interests}

The authors declare that they have no competing interests.

\section{References}

1. Lang N, Yuan HS, Wang HL, Liao J, Li M, Guo FX, Shi S and Chen ZQ: Epidemiological survey of ossification of the ligamentum flavum in thoracic spine: $\mathrm{CT}$ imaging observation of 993 cases. Eur Spine J 22: 857-862, 2013.

2. Mori K, Imai S, Kasahara T, Nishizawa K, Mimura T and Matsusue Y: Prevalence, distribution and morphology of thoracic ossification of the posterior longitudinal ligament in Japanese: Results of CT-based cross-sectional study. Spine (Phila Pa 1976) 65: 394-399, 2014.

3. Moon BJ, Kuh SU, Kim S, Kim KS, Yong EC and Dong KC: Prevalence, distribution and significance of incidental thoracic ossification of the ligamentum flavum in Korean patients with back or leg pain: MR-based cross sectional study. J Korean Neurosurg Soc 58: 112-118, 2015.

4. Ono K, Yonenobu K, Miyamoto S and Okada K: Pathology of ossification of the posterior longitudinal ligament and ligamentum flavum. Clin Orthop Relat Res 359: 18-26, 1999.

5. Wang H, Wei F, Long H, Han G, Sribastav SS, Li Z, Huang Y, Zhu R and Liang C: Surgical outcome of thoracic myelopathy caused by ossification of ligamentum flavum. J Clin Neurosci 45: 83-88, 2017.

6. Yabe Y, Honda M, Hagiwara Y, Tohjo Y, Nakajima S, Ando A, Sonofuchi $\mathrm{K}$ and Itoi E: Thoracic radiculopathy caused by ossification of the ligamentum flavum. Ups J Med Sci 118: 54-58, 2013.

7. Zhong ZM, Wu Q, Meng TT, Zhu YJ, Qu DB, Wang JX, Jiang JM, Lu KW, Zheng S and Zhu SY: Clinical outcomes after decompressive laminectomy for symptomatic ossification of ligamentum flavum at the thoracic spine. J Clin Neurosci 28: 77-81, 2016.

8. Ren L, Hu H, Sun X, Li F, Zhou JJ and Wang YM: The roles of inflammatory cytokines in the pathogenesis of ossification of ligamentum flavum. Am J Transl Res 5: 582-585, 2013.

9. Ning S, Chen Z, Fan D, Sun C, Zhang C, Zeng Y, Li W, Hou X, $\mathrm{Qu}$ X and Ma Y: Genetic differences in osteogenic differentiation potency in the thoracic ossification of the ligamentum flavum under cyclic mechanical stress. Int J Mol Med 39: 135-143, 2017.

10. Qu X, Chen Z, Fan D, Sun C, Zeng Y, Hou X and Ning S: Notch signaling pathways in human thoracic ossification of the ligamentum flavum. J Orthop Res 34: 1481-1491, 2016.

11. Hou XF, Fan DW, Sun CG and Chen ZQ: Recombinant human bone morphogenetic protein-2-induced ossification of the ligamentum flavum in rats and the associated global modification of histone H3. J Neurosurg Spine 21: 334-341, 2014.

12. Zhong ZM, Chen JT, Zhang Y, Zha DS, Lin ZS, Zhao CY, Xu JC, $\mathrm{Li} \mathrm{T}$ and Xu Z: Growth/differentiation Factor-5 induces osteogenic differentiation of human ligamentum flavum cells through activation of ERK1/2 and p38 MAPK. Cell Physiol Biochem 26: 179-186, 2010. 
13. Zhang C, Chen Z, Meng X, Li M, Zhang L and Huang A: The involvement and possible mechanism of pro-inflammatory tumor necrosis factor alpha (TNF- $\alpha$ ) in thoracic ossification of the ligamentum flavum. PLoS One 12: e0178986, 2017.

14. Park JO, Lee BH, Kang YM, Kim TH, Yoon JY, Kim H, Kwon UH, Lee KI, Lee HM and Moon SH: Inflammatory cytokines induce fibrosis and ossification of human ligamentum flavum cells. J Spinal Disord Tech 26: E6-E12, 2013.

15. Wang B, Chen Z, Meng X, Li M, Yang X and Zhang C: iTRAQ quantitative proteomic study in patients with thoracic ossification of the ligamentum flavum. Biochem Biophys Res Commun 487: 834-839, 2017.

16. Hausser $\mathrm{J}$ and Zavolan M: Identification and consequences of miRNA-target interactions-beyond repression of gene expression. Nat Rev Genet 15: 599-612, 2014.

17. Yin J, Zhuang G, Zhu Y, Hu X, Zhao H, Zhang R, Guo H, Fan X and Cao Y: MiR-615-3p inhibits the osteogenic differentiation of human lumbar ligamentum flavum cells via suppression of osteogenic regulators GDF5 and FOXO1. Cell Biol Int 41: 779-786, 2017.

18. Qu X, Chen Z, Fan D, Sun C and Yan Z: MiR-132-3p regulates the osteogenic differentiation of thoracic ligamentum flavum cells by inhibiting multiple osteogenesis-related genes. Int J Mol Sci 17: pii: E1370, 2016

19. Qu X, Chen Z, Fan D, Sun C, Yan Z, Guo Z, Qi Q and Li W: MiR-199b-5p inhibits osteogenic differentiation in ligamentum flavum cells by targeting JAG1 and modulating the Notch signalling pathway. J Cell Mol Med 21: 1159-1170, 2017.

20. Yayama T, Mori K, Okumura N, Nishizawa K, Kumagai K, Nakamura A and Imai S: Wnt signaling pathway correlates with ossification of the spinal ligament: A microRNA array and immunohistochemical study. J Orthop Sci 23: 26-31, 2018.

21. Salmena L, Poliseno L, Tay Y, Kats L and Pandolfi PP: ceRNA hypothesis: The Rosetta stone of a hidden RNA language? Cell 146: 353-358, 2011

22. Han Y, Hong Y, Li L, Li T, Zhang Z, Wang J, Xia H, Tang Y, Shi Z, Han X, et al: A Transcriptome-level study identifies changing expression profiles for ossification of the ligamentum flavum of the spine. Mol Ther Nucleic Acids 12: 872-883, 2018.

23. Ritchie ME, Phipson B, Wu D, Hu Y, Law CW, Shi W and Smyth GK: limma powers differential expression analyses for RNA-sequencing and microarray studies. Nucleic Acids Res 43 e47, 2015.

24. Larkin MA, Blackshields G, Brown NP, Chenna R, McGettigan PA, McWilliam H, Valentin F, Wallace IM, Wilm A, Lopez R, et al: Clustal W and Clustal X version 2.0. Bioinformatics 23: 2947-2948, 2007.

25. Szklarczyk D, Franceschini A, Wyder S, Forslund K, Heller D, Huerta-Cepas J, Simonovic M, Roth A, Santos A, Tsafou KP, et al: STRING v10: Protein-protein interaction networks, integrated over the tree of life. Nucleic Acids Res 43: D447-D452, 2015.

26. Kohl M, Wiese S and Warscheid B: Cytoscape: Software for visualization and analysis of biological networks. Methods Mol Biol 696: 291-303, 2011

27. Tang Y, Li M, Wang J, Pan Y and Wu FX: CytoNCA: A cytoscape plugin for centrality analysis and evaluation of protein interaction networks. Biosystems 127: 67-72, 2015.

28. Bader GD and Hogue CW: An automated method for finding molecular complexes in large protein interaction networks. BMC Bioinformatics 4: 2, 2003.

29. Dweep H and Gretz N: miRWalk2.0: A comprehensive atlas of microRNA-target interactions. Nat Methods 12: 697, 2015.

30. Li JH, Liu S, Zhou H, Qu LH and Yang JH: starBase v2.0: Decoding miRNA-ceRNA, miRNA-ncRNA and protein-RNA interaction networks from large-scale CLIP-Seq data. Nucleic Acids Res 42: D92-D97, 2014.

31. Huang DW, Sherman BT and Lempicki RA: Systematic and integrative analysis of large gene lists using DAVID bioinformatics resources. Nat Protoc 4: 44-57, 2009.

32. Dominguez E, Mauborgne A, Mallet J, Desclaux M and Pohl M: SOCS3-mediated blockade of JAK/STAT3 signaling pathway reveals its major contribution to spinal cord neuroinflammation and mechanical allodynia after peripheral nerve injury. J Neurosci 30: 5754-5766, 2010.
33. Fukushima A, Kajiya H, Izumi T, Shigeyama C, Okabe K and Anan H: Pro-inflammatory cytokines induce suppressor of cytokine signaling-3 in human periodontal ligament cells. J Endod 36: 1004-1008, 2010.

34. Chen H, Cai W, Chu ESH, Tang J, Wong CC, Wong SH, Sun W, Liang Q, Fang J, Sun Z and Yu J: Hepatic cyclooxygenase-2 overexpression induced spontaneous hepatocellular carcinoma formation in mice. Oncogene 36: 4415-4426, 2017.

35. Li D, Hao X and Song Y: Identification of the Key MicroRNAs and the miRNA-mRNA regulatory pathways in prostate cancer by bioinformatics methods. Biomed Res Int 2018: 6204128, 2018.

36. Pal J, Patil V, Mondal B, Shukla S, Hegde AS, Arivazhagan A, Santosh V and Somasundaram K: Epigenetically silenced GNG4 inhibits SDF1 $\alpha / \mathrm{CXCR} 4$ signaling in mesenchymal glioblastoma. Genes Cancer 7: 136-147, 2016.

37. Liang L, Zeng JH, Qin XG, Chen JQ, Luo DZ and Chen G: Distinguishable prognostic signatures of left- and right-sided colon cancer: A study based on sequencing data. Cell Physiol Biochem 48: 475-490, 2018.

38. Liao J, Chen Z, He Q, Liu Y and Wang J: Differential gene expression analysis and network construction of recurrent cardiovascular events. Mol Med Rep 13: 1746-1764, 2016.

39. Zhang Q, Shen Y, Jiang Y, Zhao S, Zhou D and Xu N: Overexpression of miR-182 inhibits ossification of ligamentum flavum cells by targeting NAMPT. Exp Cell Res 367: 119-131, 2018.

40. Cao Y, Wu TD, Wu H, Lang Y, Li DZ, Ni SF, Lu HB and Hu JZ: Synchrotron radiation micro-CT as a novel tool to evaluate the effect of agomir-210 in a rat spinal cord injury model. Brain Res 1655: 55-65, 2017

41. Ren D, Lin B, Zhang X, Peng Y, Ye Z, Ma Y, Liang Y, Cao L, Li X, Li R, et al: Maintenance of cancer stemness by miR-196b-5p contributes to chemoresistance of colorectal cancer cells via activating STAT3 signaling pathway. Oncotarget 8: 49807-49823, 2017.

42. Wang Y, Mao G, Lv Y, Huang Q and Wang G: MicroRNA-181b stimulates inflammation via the nuclear factor- $\mathrm{KB}$ signaling pathway in vitro. Exp Ther Med 10: 1584-1590, 2015.

43. Yamamoto K, Seike M, Takeuchi S, Soeno C, Miyanaga A, Noro R, Minegishi Y, Kubota K and Gemma A: MiR-379/411 cluster regulates IL-18 and contributes to drug resistance in malignant pleural mesothelioma. Oncol Rep 32: 2365-2372, 2014.

44. Zhao C, Wang S, Zhao Y, Du F, Wang W, Lv P and Qi L: Long noncoding RNA NEAT1 modulates cell proliferation and apoptosis by regulating miR-23a-3p/SMC1A in acute myeloid leukemia. J Cell Physiol 234: 6161-6172, 2019.

45. Meng XH, Chen XD, Greenbaum J, Zeng Q, You SL, Xiao HM, Tan LJ and Deng HW: Integration of summary data from GWAS and eQTL studies identified novel causal BMD genes with functional predictions. Bone 113: 41-48, 2018.

46. Feng F, Chen A, Huang J, Xia Q, Chen Y and Jin X: Long noncoding RNA SNHG16 contributes to the development of bladder cancer via regulating miR-98/STAT3/Wnt/ $\beta$-catenin pathway axis. J Cell Biochem 119: 9408-9418, 2018

47. Lu YF, Cai XL, Li ZZ, Lv J, Xiang YA, Chen JJ, Chen WJ, Sun WY, Liu XM and Chen JB: LncRNA SNHG16 functions as an oncogene by sponging miR-4518 and up-regulating PRMT5 expression in Glioma. Cell Physiol Biochem 45: 1975-1985, 2018

48. Yan J, Guo D, Yang S, Sun H, Wu B and Zhou D: Inhibition of miR-222-3p activity promoted osteogenic differentiation of hBMSCs by regulating Smad5-RUNX2 signal axis. Biochem Biophys Res Commun 470: 498-503, 2016.

This work is licensed under a Creative Commons Attribution-NonCommercial-NoDerivatives 4.0 International (CC BY-NC-ND 4.0) License. 